\title{
REVUE
}

\section{Arbres, arbustes, buissons et fourrages spontanés divers en régions tropicales et subtropicales (suite)}

\author{
par M. G. CURASSON
}

\section{LHIACÉES \\ Genre Asparagus}

Des espèces africaines de ce genre sont parfois broutées, surtout par les moutons et chèvres. C'est le cas pour Asparagus capensis et $A$. striatus, d'Afrique du sud, $A$. africanus et $A$. pauli-guilielmii, d'Afrique Occidentale.

\section{Genre Asphodelus}

Asphodelus microcarpus est une Liliacée de la zcne maritime, en Lybie, dont les feuilles sont mangées.

\section{Genre Astelia}

Astelia nervosa forme en Nouvelle-Zélande des peuplements importants dans les régions forestières basses; les feuilles, très grandes, sont entièrement consommées par le bétail.

\section{Genre Muscari}

Muscari maritimum est mangée dans les steppes de la zone maritime de Tripolitaine.

\section{LOGANIACÉES}

Chilianthus arboreus, arbre d'Afrique du Sud, fournit des feuilles comestibles dont la composition est la suivante : eau, 40,5; protéine brute, 8,9 ; extrait éthéré, 8,9; cellulose brute, 18,4; extractif non azoté, 60 ; cendres 4,4 .

\section{LORANTHACÉES}

\section{Genre Loranthus}

Les espèces de ce genre, qui parasitent divers arbres, sont des plantes succulentes que mangent volontiers les animaux. Les principales sont : Loranthus acaciae, d'Afrique du Nord, Soudan Égyptien, Érythrée, L. chevalieri, du Soudan Français, qui pourrait être dangereux, L. globiferus, du Sud saharien, $L$. pendulus, d'Australie.

\section{MALVACEES}

Genre Abutilon

Plusicurs espèces sont mangées par lo bétail A. fruticosum, A. muticum, A. mauritanicum, d'Afrique Occidentale, et $A$. incanum, d'Amérique (Texas). Cette demière contient $19,5 \%$ de protéine, $1,8 \%$ d'extrait éthéré, $22,5 \%$ de cellulose et $46,3 \%$ d'extractif non azoté.

\section{Genre Corchurus}

Corchurus antichorus est une herbe mangée par le chameau dans l'Inde, en Mauritanie, C. trilocularis, du Queensland, est également mangée.

\section{Genre Gossypium}

Les divers Gossypium qui fournissent le coton ont des feuilles que les animaux consomment volontiers. Le coton indigène (Gossypium punctatum) est cultivé depuis longtemps au Congo Belge comme arbuste fourrager; sa végétation est pérenne; je l'ai essayé avec succès dans le Sahel; il se contente de terres moins fraîches que l'ambrevade et, lorsqu'il est taillé, il peut demeurer une dizaine d'années sur le même terrain; ses feuilles et ses capsules sont bien acceptees par tous les animaux, bovins compris. C'est un arbuste très intéressant à cultiver mais qui, dans le Sahel, ne vient qu'à proximité des mares.

Les feuilles des autres cotonniers cultivés en Afrique Occidentale sont volontiers mangées par les animaux; c'est le cas pour l'espèce indigène G. obtusifolium, ainsi que celles qu'amenèrent les conquérants islamiques, G. purpurascens deserticum, qui cèdent d'ailleurs peu à peu la place aux cotonniers importés en zones irriguées; dans le Sud, on trouve G. barbabense maritimum, G. barbadense vitifolium, G. peruvianum, et divers hybrides.

\section{Genre Hibiscus}

Les feuilles d'Hibiscus esculentus, ainsi que les graines, sont mangées (Texas); de même pour 
H. sabdoriffa, de l'Inde, d'Amérique du Sud (Chili), d'Afrique Occidentale. Cette malvacée est cultivée à peu près dans toutes les zones tropicales; les pièces du calice, épaisses et colorées en rouge, sont utilisées par les indigènes comme condiment. Les feuilles que mange le bétail sont grandes et veloutées; elles sont utilisées comme légume (oseille de Guinée). Ira

\section{Genre Sida}

Des espèces buissonneuses de ce genre sont mangées, surtout par les moutons. Sida corrigata (= pedunculata) d'Australie, S. rhombifolia, d'Uruguay, Venezuela, $S$. cordifolia, $S$. carpinifolia, d'Afrique Occidentale, S. grewioïdes, d'Afrique Orientale.

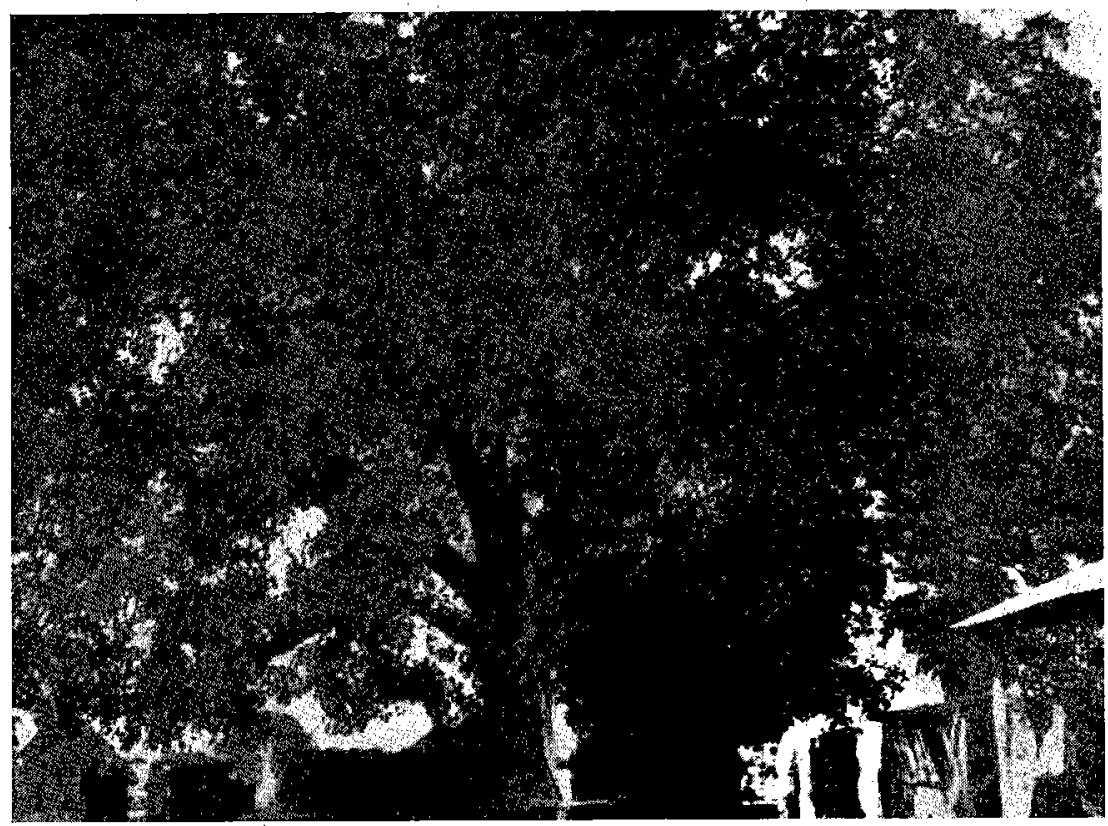

Cliché Adam. Collection C.T.F.T.

Fig. 1. - Ficus (A.O.F.).

présence d'acide citrique en quantité parfois importante doit en limiter l'emploi.

Les feuilles d'Hibiscus cannabinus, $H$. quinquelobus, deux espèces textiles, sont également mangées en Afrique Occidentale, ainsi que celles d' $I$. micranthus, d'Afrique Orientale.

\section{Genre Hoheria}

Les arbustes de ce genre, Hoheria populnea, H. glabrata, sont des espèces fourragères médiocres de Nouvelle-Zélande (zones assez froides).

\section{Genre Malva}

Diverses espèces herbacées du genre sont mangées dans le Sahara. De même Malva parviflora de l'Inde, $M$. sylvestris qui est vendue sur les marchés de Libye, de Tripolitaine.

\section{Genre Malvaviscus}

Malvaviscus arboreus forme, au Chili, des buissons que mange volontiers le bétail: La plante est très résistante à la sécheresse.

\section{MARATTIACÉES \\ Genre Marattia}

Une grande fougère de Nouvelle-Zélande, Marattia fraxina, possède de volumineux rhizomes, nourriture favorite des Maoris et que les porcs recherchent.

\section{MÉLIACÉES \\ Genre Dysoxylum}

Dysoxylum spectabilis est un grand arbre d'Australie, de Nouvelle-Zélande, dont les fruits son comestibles.

\section{Genre Khaya}

Les feuilles de Khaya senegalensis, le cail-cedrat, sont parfois distribuées aux ruminants en Afrique Occidentale.

\section{Genre Melia}

Melia azedarrach, de l'Inde, largement introduit en Afrique, a des feuilles qui sont parfois distribuées. 
La plante peut être toxique. Ises feuilles sont utilisées pour des infusions ou extraits qui tuent les sauterelles.

\section{Genre Nymonia}

Les moutons mangent volontiers les feuilles de Nymonia capensis, d'Afrique du Sud.

\section{Genre Ptaeroxylon}

Dans la province du Cap, Ptaeroxylon utile est considérée comme arbre fourrager.

\section{Genre Turraeanthus}

Les fruits de Turraeanthus africana, d'Afrique Occidentale, sont mangés par les animaux quand ils sont tombés. Cependant l'écorce serait toxique.

\section{MOLLUGINACÉES}

\section{Genre Gisekia}

Gisekia pharnacoïdes, du Sud saharien, est un bon fourrage pour le chameau.

\section{MORACÉES}

\section{Genre Antiaris}

Les graines d'Antiaris atricana sont mangées par les chères et les feuilles par le chameau; cependant on considère, au Dahomey, les graines comme toxiques.

\section{Genre Artocarpus}

Le jacquier Artocarpus integrifolia, l'arbre à pain, $A$. incisus ont des feulles et surtout des fruits qui sont recherchés avidement par les animaux, ou qu'on leur distribue aux Antilles, à Ceylan. De même les fruits d'A. nobilis. On retrouve $A$. incisus et $A$. integrifolia au Mexique. au Cuatemala, ainsi qu'A. communis.

\section{Genre Ficus}

Le feuillage et les fruits de plusieurs espèces de figuiers sauvages sont appréciés des animaux.

En Afrique Occidentale, certaines espèces sont considérées comme galactagogues; c'est à ce titre que les Peulhs donnent aux vaches les feuilles et fruits de Ficus capensis. En Guinée Française, les indigènes distribuent feuilles et fruits de $F$. maclaudi aux vaches, juments, brebis et chèvres. D'après des essais de de Goldfiem (1935) qui demandent confirmation, la plante serait effectivement galactagogue. Sont également utilisés les feuilles et les fruits tombés à terre, de F. toro, F. graphalocarpa, F. platiphylla.

Dans l'Est de Madagascar, on distribue le feuillage de $F$. caecutifolia.

Au Soudan Egyptien, on utilise les feuilles de $F$. benghalensis, $F$. religiosa, introduits comme arbres d'ombrage, pour les chèvres.

Au Tanganyika, on distribue les feuilles de F. sycomorus.

En Afrique du Sud, les fruits de $F$. soldanella sont mangés.

En Australie on a recours à F. rubiginosa, F. watkinsonia, F. macrophylla. Les feuilles tombées de cette dernière espèce sont surtout distribuées aux vaches laitières. Au Queensland on considère F. opposita comme excellent.

En Amérique du Sud, F. benjamina introduit au Brésil a des feuilles particulièrement intéressantes en saison seche; de même $F$. padifolia (Guatémala).

Dans l'Inde toutes les espèces sont 'utilisées sauf F. elastica. Certaines sont cultivées parfois dans les villages pour la production de fourrage. On peut citer: F. hookeri, F. hispida, F. nemoralis, F. roxburGhii, $F$. benghalensis, $F$. religiosa, $F$. glabella, F. rumphii, F. glomerata, F. infectoria, F. retusa.

La valeur nutritive des feuilles de figuiers est indiquee par la composition des feuilles de $F$. carica (Maymone et Petrucci 1943) : Eau, 67,6; protéine brute, 4,3 ; protéine vraie, 4 ; graine, 1,7 ; cellulose, 4,7 ; cendres, 5,3 .

\section{Genre Morus}

Morus alba et $M$. nigra, le mûrier blanc et le mûrier noir, sont utilisés en certaines régions non seulement comme plantes séricicoles, mais aussi comme arbres fourragers. En Afrique du Nord, dans le bassin méditerranéen, le múrier fournit en été, sans irrigation, un fourrage très apprécié, riche en protéines et graisses, et pouvant remplacer les légumineuses. On l'utilise à cet effet en France, en Algérie et au Maroc, et à Chypre.

Les feuilles ont la composition suivante, comparée à celle de l'herbe de prairie et au sainfoin (d'après Velu) :

\begin{tabular}{|c|c|c|c|c|}
\hline & M.A. & M.G. & H. de C. & CELLULOOSE \\
\hline Feuilles de mûrier fraîches & 5,5 & 2,4 & 15,5 & 2,91 \\
\hline - sèches . & 16,3 & 4,1 & 49,6 & 6.9 \\
\hline Herbe de prairie $\ldots \ldots \ldots \ldots \ldots \ldots \ldots \ldots \ldots$ & 3 & 0,8 & 13,1 & 6,0 \\
\hline Sainfoin $\ldots \ldots \ldots \ldots$ & 3,5 & 0,7 & 7,6 & 6,9 \\
\hline
\end{tabular}


On" considère que $150 \mathrm{~kg}$ de feuilles fraîches, correspondent à $100 \mathrm{~kg}$ de bon foin. En France, on donine 7 à $10 \mathrm{~kg}$ aux bovins.

On donne les feuilles vertes, sèches ou ensilées; elles sont mangées sous l'arbre alors qu'elles tombent à l'automne, encore vertes ou commençant à jaunir. Pour les faire sécher on les met en lits de 20 à 30 centimètres qu'on brasse de temps en temps. Pour l'ensilage, il faut charger lourdement et ajouter $1 \mathrm{~kg}$ de sel par $100 \mathrm{~kg}$ de feuilles. On fait toutes ces opérations au moment où, à l'approche de l'automne, l'élevage des vers à soie est sur le point d'ètre terminé. On peut aussi distribuer aux animaux les tiges feuillées qu'on a coupées. On peut faire 4 ou 5 coupes dans l'année.

En Asie, on cultive parfois $M$. alba et M. multicaulis en lignes rapprochées de 50 centimètres, et on coupe plusieurs fois par an. On obtient ainsi une sorte de prairie.

D'autres espèces du genre Morus sont comestibles. C'est le cas pour' $M$. mesozygia d'Afrique Occidentale, M. microphylla, du Texas.

\section{Genre Paratrophis}

Les feuilles de Paratrophis microphylla, petit arbre de Nouvelle-Zélande sont mangées.

\section{Genre Trophis}

Trophis americana arbre de la savane vénézuélienne, a des feuilles mangées par le bétail.

\section{MORINGACÉES \\ Genre Moringa}

Moringa pterygosperma $(=M$, oleifera), le "Neverdié », "Nevredaye » de l'Inde, introduit en Afrique, dont la graine est oléagineuse, a des feuilles que les indigènes distribuent parfois ; elles pourraient être cyanogénétiques. $M$. concanensis de l'Inde, a également des pousses et des feuilles comestibles comme $M$. aptera, d'Égypte dont les graines sont aussi oléagineuses.

\section{MUSACÉES \\ Genre Musa}

Les feuilles du bananier renferment $1,7 \%$ de matières azotées et $5,2 \%$ de graisse; une unité fourragère est constituée par $7 \mathrm{~kg}$ de feuilles. Le tronc est moins riche en matières azotées et en graisses (traces). Il faut $12 \mathrm{~kg}$ pour une U.F. (Rémond et Winter 1944).

\section{MYOPORACÉES \\ Genre Eremophila}

D'assez nombreuses espèces du genre existent en Australie; ce sont des arbres, arbustes ou buissons. E. bignoniiflora, E. longifolia, sont considérés comme d'excellents fourrages.
De même E. maculata; mais cette dernière cspc̀co pourrait être fortement cyanogénétique. $E$. latrobei, $E$. goodivinii, E. bowmani, sont des arbres du Queesland; $E$. divaritaca, E. polyclada, E'. glabra, des buissons de l'État de 'Victoria, de faible valeur fourragère. Dans l'Australie Occidentale, on trouve E. oppositifolia, E. scoparia.

\section{Genre Myoporum}

Plusieurs arbres d'Australie, de Nouvelle-Zélande, sont comestibles ou dangereux. Myoporum platycarpum est souvent distribué et semble un bon fourrage. Par contre, $M$. deserti est toxique; les animaux le délaissent habituellement. $M$. laetum est également dangereux (Aston); les baies et les fruits surtout ont un effet drastique et toxique (Webster). M. montanum est de valeur douteuse.

\section{MYRSINACÉES}

Genre Reptonia

Reptonia buxifolia, de l'Inde, est un buisson épineux toujours vert dont les feuilles sont surtout mangées par le chameau.

\section{Genre Suttonia}

Un ar buste de Nouvelle-Zélande, Sullonia ausltalis, est volontiers mangé par le bétail. De même $S$. salicina.

\section{NYCTAGINACÉES \\ Genre Borhavia}

Plusieurs espèces du genre constituent de bons fourrages quoique peu abondants. Bœrhavia repens pousse sur les dunes (Indes) et est mangée par les chèvres, les moutons, le chameau. La graine: de $B$. diffusa est mangée par les indigènes, $B$. tenuifolia est une espèce du Texas. B. verticillata peut former d'assez grandes étendues dans le Sahel, dans le Sahara, où elle constitue des pâturages de printemps, pour le chameau; on retrouve' dans le Sahel B. repens, avec $B$. diffusa, $B$. viscosa.

\section{Genre Phæoptilum}

Phroptilum spinosum est une plante buissonneuse d'Afrique du Sud très recherchée des moutons.

\section{OLACACÉES \\ Genre Ximenia}

Les fruits de Ximenia caffra sont mangés en Afrique du Sud. De même ceux de $X$, americana (olive sauvage) d'Afrique du Sud, Afrique Orientale et Occidentale.

\section{OLÉACÉES Genre Linociera}

Les feuilles de Linociera nilotica d'Afrique Occidentale seraient comestibles. 


\section{Genre Olea}

Olea europea, l'olivier commun, offre ses feuilles et différents dérivés de ses fruits. Les feuilles et rameaux sont distribués aux animaux dans la région méditerranéenne. Les feuilles ont la composition suivante : protéine brute, 13,3 ; extrait éthéré, 6,3 ; cellulose brute, 33,1; extractif non azoté, 42,5; cendres, 4,7 . On les donne à raison de $3-4 \mathrm{~kg}$ pour les
Olea fragrantissima, O. lineata, O. odorata $c=0$. europea), O. virgata, O. lanceolata, sont des aroustes de Nouvelle-Zélande, dont les moutons ef les bovins mangent volontiers les feuilles. O. lanceolata a été accusé de causer des troubles, mais' ce ñ'est pas prouvé. $O$. cuspidata est une espèce de l'Inde (Baluchistan) que mangent les bovins, mais slirtout les chameaux.

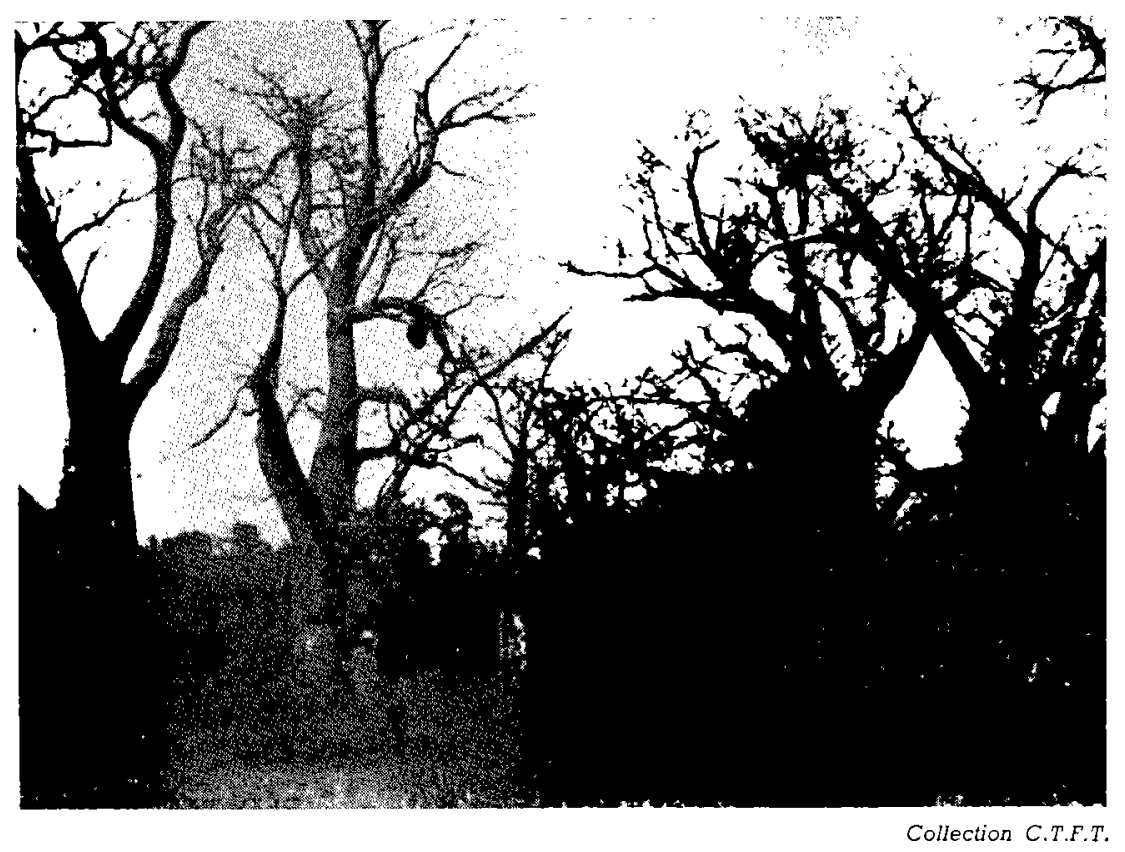

Fig. 2. - Forêt sénégalaise comportant de nombreuses espèces : Dichrostachys glomerata, Grevia bicolor, Pterocarpus erinaceus, baobabs, caill-cedrats, etc.

bovins, $0,500 \mathrm{~kg}$ à $1 \mathrm{~kg}$ pour les moutons. On peut les faire sécher, les conserver en poudre. Un bon procédé consiste à distribuer les feuilles sèches après immersion dans l'eau salée. On donne $0,750 \mathrm{~kg}$ à $1 \mathrm{~kg}$ de feuilles sèches par $100 \mathrm{~kg}$ de poids vif.

Les feuilles fraiches ont la composition suivante: eau, 42,1; protéine brute, 7,6 ; extrait éthéré, 4,1 ; extractif non azoté, 32,3 ; cellulose, 10,3; cendres, 3,5 .

La teneur en vitamines est : Vitamine C, $38 \mathrm{mg}$, Vitamine $\mathrm{E}, 6,8 \mathrm{mg}$, carotène, $0,36 \mathrm{mg}$.

Olea africana ( $=O$. verrucosa. Var. africana) est une espèce d'Afrique du Sud dont les feuilles sont mangées avidement, là où les moutons et bovins la rencontrent; on est obligé de la protéger. Les feuilles ont la composition suivante : protéine brute, 10,2; extrait éthéré, 8,8 ; cellulose brute, 22,5 ; extractif non azoté, 51,1 ; cendres, 7,4 .

\section{OMBELLIFÈRES}

\section{Genre Aciphylla}

Plusieurs espèces du genre forment en NouvelleZélande des étendues plus ou moins grandes, compactes, de plantes épineuses que mange cependant le mouton; les principales sont : Aciphylla colensoi et $A$. squarrosa.

\section{Genre Deverra}

Deverra chloranta, D. scoparia, du Sahara d'Afrique du Nord, sont mangées par le chameau: leur pollen causerait des ophtalmies.

\section{Genre Pituranthos}

En Tripolitaine, Cyrénaïque, Pituranthos chioranthus, $P$. scoparius, $P$. tortuosus sont un assez bon fourrage pour le chameau. 


\section{OROBRANCACÉES}

\section{Genre Phelypaa}

Phelypæa violacea; en Tripolitaine, Cyrénaïque, parasite généralement les Haloxylon. Le chameau la mange volontiers. Elle est recherchée des gazelles à la viande desquelles elle donnerait un goûtagréable.

\section{OXALIDACEES}

\section{Genre Oxalis}

De nombreuses espèces de ce genre existent en Afrique, Amérique, Australie, et peuvent être mangées, par le mouton surtout. Leur teneur en acide oxalique fait quiune consommation prolongée peut être dangereuse. Cela a été montré pour O. cernua, espèce australienne et $O$. corniculata, espèce africaine. En Amérique du Sud, daris les régions subtempérées, on considère comme comestibles : O. articulata, O. macachin.

\section{PALMIERS}

\section{Genre Acrocomia}

Acrocomia totai est un palmier des zones buissonneuses du Paraguay. Les bovins sont mis à pâturer dans les régions où il existe, pour en manger les fruits; on les ramene ensuite au corral où les amandes, rejetées avec les excréments, et lavées par les pluies, sont ramassées pour en extraire l'huile.

Au Brésil, à Costa-Rica, on donne au bétail, et particulièrement aux vaches laitières, les feuilles d'A. sclerocarpa. On a dû réglementer la coupe de ce palmier à Costa-Rica.

\section{Genre Attalea}

Les palmiers de ce genre, Attalea phalerata, A. princeps du Brésil, ont des feuilles comestibles. Celles du premier sont considérées comme un excellent fourrage; celles du second sont données aux chevaux.

\section{Genre Borassus}

Borassus flabellifer (B. aethiopicum), espèce de la région soudanaise (ronier), de l'Inde (Ceylan) a des feuilles dures qui sont parfois mangées.

\section{Genre Cocos}

Les feuilles de Cocos nucifera, quańd elles sont jeunes, sont parfois utilisées comme fourrage.

\section{Genre Copernicia}

Les palmiers de ce genre sont très répandus dans les savanes du Venezuela, de Colombie; les feuilles de C. tectorum, de C. cerifera (Brésil) sont mangées par le bétail.

\section{Genre Hyphæne}

Hyphæne thebaïca, le palmier doum, répandu dans toute l'Afrique sahélienne, fournit les feuilles de la couronne qui sont distribuées en saison sèche; l'enveloppe des noix est souvent donnée aux ânes, et la graine aux moutons après avoir été rôtie et concassée au mortier.

\section{Genre Phenix}

Les feuilles de Phenix dactilifera constituent un fourrage de peu de valeur.

\section{Genre Syagrus}

Syagrus oleracea, S. picrophylla sont des palmiers du Brésil, dont les feuilles sont mangées; les fruits du dernier sonl également mangés.

\section{PANDANACÉES \\ Genre Freycinetia}

Freycinetia banksii est une liane de NouvelleZélande dont les fruits, abondants et succulents, sont mangés par le bétail.

\section{PARONYCHIÉES \\ Genre Herniaria}

Herniaria hirsuta, qu'on rencontre dans la région méditerranéenne et l'Inde (Baluchistan), est mangée par les chameaux et les chèvres.

\section{PÉDALIACÉES \\ Genre Sesamum}

Sesamunn indicum, originaire de I'Inde, est cultivée dans beaucoup de régions tropicales; il en existe de nombreuses variétés. Les graines servent à la fabrication de l'huile de sésame. Le tourteau est un excellent aliment.

\section{PHYTOLACCACÉES \\ Genre Codonocarpus}

Codonocarpus cotinifolia est une espèce australienne répandue, que le chameau mange volontiers.

\section{Genre Phytolacca}

Phytolacca dodecandra (= dioica ?) a des feuilles que les indigènes de la: Guinée trançaise distribuent comme galactagogue. P. dioica, originaire d'Amérique du Sud, est considérée en Australie comme un arbre fourrager intéressant; il pousse très vite et la taille, qui favorise la croissance, donne un fourrage que le bétail mange volontiers; par contre, $P$. octandra pourrait être dangereux. $P$. acinosa est mangée par le chameau dans l'Inde.

Le genre est aussi représenlé en Afrique du Sud. 


\section{PITTOSPORACÉES}

Genre Pittosporum

Pittosporum phillyracol̈des est un arbre très répandu en Australie, où il est considéré comme un bon fourrage. De même $P$. tenuifolium, utilisé en Nouvelle-Zélande pour former des haies. Cependant. d'après Allen (1944) le bétail les estime peu.

\section{PLantaginacés \\ Genre Plantago}

Plantago albicans est mangé par les moutons sur les terrains sablonneux de Tripolitaine. Il ressemble à $P$. ovata, du Sud algérien, également mangé.

\section{PLOMBÁGINACÉES \\ Genre Bubania}

Bubania feei est une herbe salée du Sahara, que le chameau mange en saison des pluies.

\section{Genre Limoniastrum}

Limoniastrum guyonianum est mangée par le chameau, dans les steppes de l'intérieur de Tripolitaine. On la retrouve dans les terres salées du Sahara. L. monopetalum est une espèce voisine.

\section{Genre Plumbago}

Plumbago capensis, d'Afrique du Sud, est considérée comme fourragère.

\section{Cenre Statice}

Des especes de ce genre sont des herbes salées recherchées du chameau : Statice bonduellii, S. globularidefolia, S. pruinusa, S. thonini, S. tubiferia. On les rencontre dans le Sahara, en Lybie, Tripolitaine, en Somalie

\section{POLYGALARCÉES}

\section{Genre Muraltia}

Muraltia depressa et $M$. salsolacea, d'Afrique du Sud, sont mangées volontiers.

\section{Genre Polygala}

Polygala asbestina, $P$. leptoloba, $P$. hottentota, d'Afrique du Sud, sont recherchées des moutons, $P$. baikei est une espèce d'Afrique Occidentale. Plusieurs espèces sud-américaines sont mangées : $P$. australis, $P$. linoïdes, etc.

\section{Genre Securidata}

Les feuilles de Securidata longipedunculata d'Afrique Occidentale, sont comestibles el parfois distribuées.

\section{POLYGONACÉES \\ Genre Calligonum}

Calligonum comosum, du Sahara, de l'Inde, est un arbuste précieux pour le chameau qui mange aussi C. caput medusae (Asie Centrale), C. mongolicum (Mongolie), C. polygonoïdes (Inde).

\section{Genre Imex}

Imex spinosa est une bonne espèce pour le chameau, parfois cultivée en Cyrénaïque.

\section{Genre Muehlenbeckia}

Muehlenbeckia australis, $M$. complexa, $M$. cunninghamii, sont des lianes australiennes que mange le bétail.

\section{Genre Polygonum}

Polygonum aviculare, des régions tempérées, réussit aussi en régions subtropicales; en certaines régions d'Amérique du Sud, il envahit les luzernières. Des espèces voisines, qui peuvent être buissonneuses, sont mangées par les moutons et les chèvres : P. glabrum, d'Afrique Occidentale, $P$. bowenkampi, du Chili, $P$. persicarioides, du Pérou.

\section{Genre Pterococcus}

Les pousses de Pterococcus aphylla sont mangées par le chameau en Mongolie.

\section{POLYPODIACEES \\ Genre Cheilantes}

Des fougères herbacées de ce genre sont mangées, à défaut d'herbe, par le bétail en Australie; une consommation abusive pourrait causer des accidents.

\section{Genre Notholcena}

Notholcena distans est également une fougère australienne habituellement délaissée du bétail, qui s'y attaque cependant parfois; la consommation pourrait causer des accidents de "vertige ».

\section{PORTULACACÉS}

\section{Genre Portulacaria}

Les feuilles de Portulacaria afra sont mangées en Afrique du Sud.

\section{Genre Talinum}

Talinum cafrum est un arbuste d'Afrique du Sud dont les feuilles sont mangées.

\section{RENONCULACÉES}

Genre Clematis

Diverses espèces sont mangées (Clematis drummondii, du Texas); d'autres sont toxiques (Afrique Occidentale, Amérique du Sud.) 


\section{Genre Delphinium}

Delphinium nanum, est mangée par le chameau en Cyrénaïque.

\section{RÉSÉDACÉES \\ Genre Oligomeris}

Oligomeris capensis, des terrains acides d'Afrique du Sud, est broutée, plus volontiers que O. dregeana, des zones sablonneuses. de la dernière ont la composition suivante : protéine brute 17,4; extrait éthéré, 4,5; cellulose brute, 24,2; extractif non azoté, 49,2; cendres, 4,8.

\section{Genre Zizyphus}

Les jujubiers sont recherchés, surtout des moutons et des chèvres, pour leurs feuilles et leurs fruits. Ils sont également intéressants en raison de leur résistance à la sécheresse. Les principales espèces sont : Zizyphus jujuba (Afrique du Nord, Afrique Occiden-

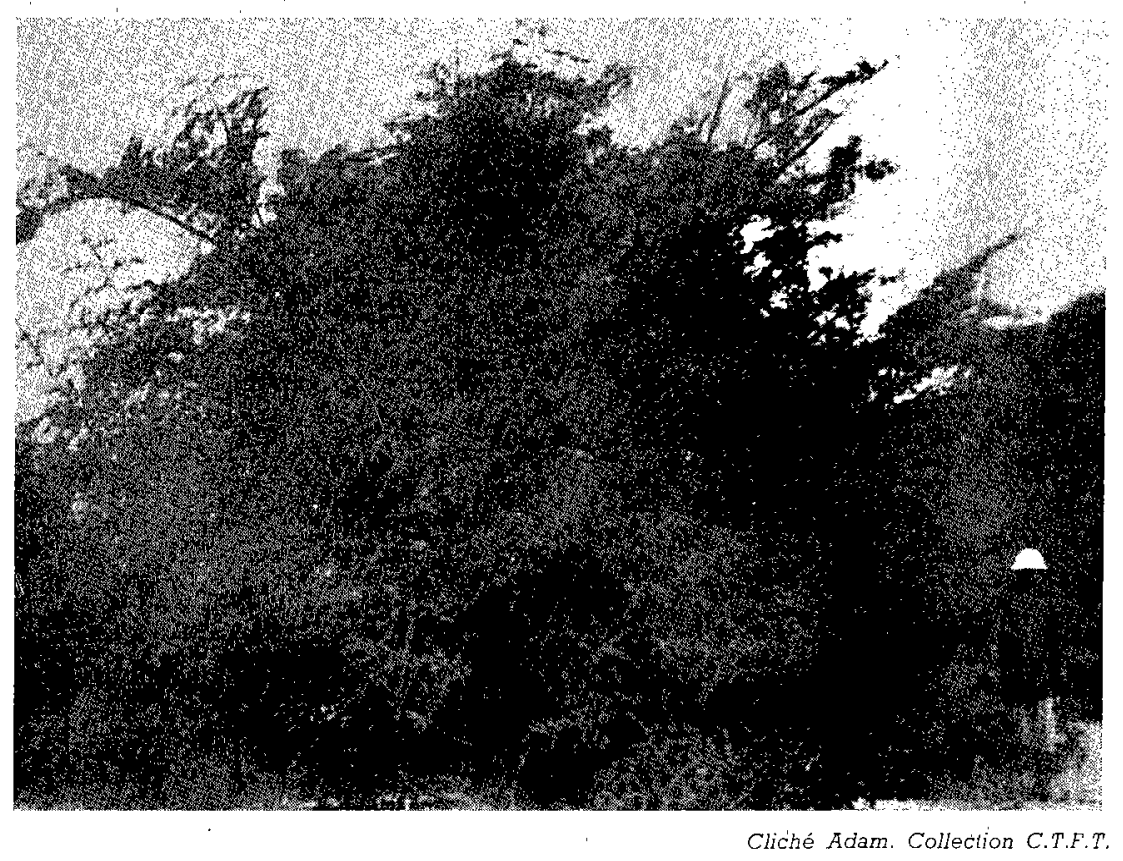

Fig. 3. - Zizyphus jujuba (A.O.F.).

\section{RHAMNACÉES}

Genre Discaria

Discaria toumatæe est un arbuste épineux de Nouvelle-Zélande que mangent les bovins et les chevaux.

\section{Genre Phylica}

Les feuilles de Phylica oleaefolia d'Afrique du Sud, sont mangées volontiers.

\section{Genre Pomaderris}

Pomaderris apetala est un arbuste de NouvelleZélande, parfois cultivé, très recherché du bétail.

\section{Genre Rhamnus}

Les espèces du genre constituent des arbustes que mangent surtout les petits ruminants : Rhamnus persica dans l'Inde (Baluchistan), en Asie Centrale, $R h$. californica en Amérique du Nord. Les feuilles tale, Indes), Z. joazeiro (Brésil), Z. mistol (Argentine), $Z$. pseudo-jujuba (Sahel), Z. mucronata (Sahel, Afrique du Sud), $Z$, lotus (Sahara, Tripolitaine), $Z$. nummularia (Indes), Z. saharae (Sahara), Z. spinachristi (Sahara, Arabie), Z. xylopyrus (Indes). Les fruits de $Z$. mucronata sont toxiques.

La valeur alimentaire des feuilles des jujubiers est en général assez grande; au Brésil, $Z$. joazeiro aurait une valeur égale à celle de la luzerne; dans certaines régions de l'Inde, on récolte les feuilles de $Z$. jujuba et $Z$. nummularia qu'on considère comme excellentes pour les vaches laitières.

\section{ROSACÉES Genre Neurada}

Neurada procumbens du Sahara, du Soudan égyptien, est un bon pâturage à chameau, après les pluies. 


\section{Genre Parinarium}

Plusieurs arbres africains de ce genre sont intéressants par leurs fruits.

\section{Genre Photinia}

Photinia serrulata, petit arbuste asiatique, introduit en Amérique, aux Philippines, a des feuilles assez coriaces que mangent cependant les chevres. Les feuilles jeunes peuvent être cyanogénétiques.

\section{Genre Poterium}

La pimprenelle, Poterium sanguisorba est une rosacée vivace qui pourrait être intéressante dans les régions subtropicales. Elle est spontanée en divers endroits d'Afrique du Nord. En Europe, les animaux la délaissent généralement. Elle est cependant utilisée en Australie et en Afrique australe, car elle s'est montrée très résistante au froid, à la sécheresse, au piétinement, au pâturage intensif. Son système Iadiculaire, pivotant comme celui de la luzerne, lui permet de rester verte fort longtemps. On l'emploie surtout dans les sols secs, pauvres et pierreux. Elle n'a pas réussi au Soudan Français, en zone sahélienne.

\section{Genre Prunus}

Ce genre est représenté par plusieurs espèces fourragc̀res dans les pays chauds. Dans l'Inde, on rencontre Prunus eburnea, $P$. acuminata. Les espèces américaines $P$. maritima, $P$. minutiflora (Texas) fournissent feuilles et fruits.

\section{Genre Rubus}

Une liane de Nouvelle-Zélande, Rubus australis, est mangée par les chèvres et les moutons, ainsi que R. subpauperatus.

\section{RUBIACÉES}

Genre Adina

Les feuilles d'Adina cordifolia, de l'Inde sont comestibles. Celles d'A. microcephala, d'Afrique occidentale le seraient aussi.

\section{Genre Anthospermum}

Anthospermum ciliare des terrains acides d'Afrique du Sud est mangée par les ruminants. A. rigidum est bien appétée, ainsi que $R$. trichostomum.

\section{Genre Canthium}

Canthium oleifolium est un arbre d'Australie, dont les feuilles sont considérées comme un excellent fourrage. Elles ont la composition suivante : protéine brute : 10,2; extrait éthéré, 4,4; cellulose brute, 19,1; extractif non azoté, 59,1; cendres, 7,2 .

Les feuilles de plusieurs espèces africaines dénommées "citronnier de forêt », dont $C$. discolor, C. venosum auraient des feuilles toxiques.

\section{Genre Carpococe}

Diverses espèces du genre sont mangées sur les terrains acides d'Afrique du Sud.

\section{Genre Coprosma}

Des espèces du genre sont assez nombreuses en Nouvelle-Zélande. Quelques-unes sont mangées : Coprosma grandifolia, C. robusta, C, tenuifolia.

\section{Genre Feretia}

Feretia canthioïdes, d'Afrique Occidentale, est un bon fourrage.

\section{Genre Gardenia}

Des arbustes ou arbres africains constituent, en Afrique Occidentale, dans la zone sal,élienne et soudaraise, des espèces dont certaines sont recherchées avidement du bétail. En Nigéria, en Gold Coast, on signale Gardenia aquala, G. sokolensis; au Soudan Francais G. erubescens, G.triacantha, G.jovis tonantis. Le genre est également représenté dans l'Inde par plusieurs espèces fourragères.

\section{Genre Mitragyna}

Mitragyna inermis, d'Afrique Occidentale, a des feuilles appréciées.

\section{Genre Nenax}

Nenax microphylla est très recherchée sur les terrains salés d'Afrique du Sud, à tel point que l'espèce tend à disparaître en certaines régions. $N$. dregei, des mêmes régions, est également consommé.

\section{RUTACÉS \\ Genre Acmadenia}

Acmadenia uniflora est un buisson volontiers mangé en Afrique du Sud.

\section{Genre Agathosma}

Agathosma scaberula est mangée par le mouton en Afrique du Sud.

\section{Genre Diosma}

Diosma aspalathoïdes, D. guthrieæ sont des buissons fourragers d'Afrique du Sud.

\section{Genre Fagara}

Les feuilles de plusieurs arbres de ce genre sont mangées volontiers : Fagara chalybæa d'Afrique Orientale, F. xanthoxylödes, d'Afrique Occidentale.

\section{Genre Haphophyllum}

Haphophyllum vermiculare est mangée par le chameau, et utilisée contre les coliques en Libye, Tripolitaine. 


\section{Genre Paganum}

Paganum harmata, plante vivace du Sud algérien et tunisien, est parfois mangée malgré son odeur forte, surtout par le chameau. On pourrait observer des accidents toxiques.

\section{Genre Ruta}

Ruta bracteosa est mangée en Tripolitaine (région de collines).

\section{SALICACÉES}

\section{Genre Populus}

Les peupliers comme les saules, sont des arbres des zones tempérées et subtropicales. Cependant Populus euphratica en Asie Mineure et notamment en Irak fournit des feuilles que mangent volontiers les animaux, particulièrement le chameau.

\section{Genre Salix}

T.es arbres de ce genre se rencontrent dans les régions tempérées ou subtropicales. Salix babylonica, utilisé comme arbre ornemental en Australie, Nouvelle-Zélanıde, en Amérique du Sud, a une certaine valeur fourragère. Les feuilles tombées sont mangées par le mouton. On utilise aussi les feuilles de $S$. alba, S. caprea, S. fragilis, S. viminalis, on élague ces arbres à cet effet.

Au Transvaal on distribue les feuilles de $S$. capensis qui renferment $0,6 \%$ de P2 O5 alors que les herbes des mêmes régions n'en renferment que $0,05 \%$ (Honrici 1944).

Diverses espèces des régions semi-désertiques (toundras) de l'U.R.S.S. sont fourragères.

\section{SALVADORACÉES}

\section{Genre Salvadora}

Salvadora persica est un arbuste de l'Afrique sahélienne, de l'Inde, dont les feuilles et fruits sont mangés par tous les ruminants: Les indigènes d'Afrique Occidentale le disent galactogène. Il donne au lait une odeur et une saveur spéciales. S. indica, S. oleoïdes, de l'Inde, sont également consommés. S. oleoïdes peut causer de la diarrhée chez les chameaux qui le consomment.

\section{SALVINIACÉES}

\section{Genre Azolla}

Les plantes aquatiques de ce genre particulierement Azolla pinnata sont utilisées en Indochine comme fourrage et engrais vert. Elles servent à la nourriture des porcs et des canards! C'est un aliment très aqueux et de végétation peu abondante. Voici une analyse d'Azolle non' cultivée : eau, 93,13; azote, 4,$2 ; P_{2} O_{5}, 0,931$; poldsse, 3,18; chaux, 3,5.

\section{SATOLACÉES \\ Genre Oxyris}

Oxyris abyssinica, arbuste tannifère qui forme de grands peuplements en Érythrée est apprécié du chameau. La consommation colore l'urine et les fèces en rouge.

\section{Genre Thesium}

Thesium hystrix du Karoo d'Afrique du Sud, pratiquement sans feuilles, est un assez bon fourrage.

\section{SAPINDACÉES \\ Genre Atalaya}

Atalaya hemiglauca est un arbre très répandu en Australie; il est considéré comme donnant un excellent fourrage; mais sa teneur en saponine, variable selon les régions, fait qu'il peut être dangereux.

\section{Genre Blighia}

Parmi les petits arbres africains do co genre, Blighia sapida a des feuilles que mangent les moutons et les chevaux, mais qui pourraient être dangereuses. Le fruit est comestible, mais la graine serait toxique.

\section{Genre Dodonea}

Des arbustes de ce genre existent en Australie, Afrique du Sud. Dodonea attenuata (Australie) a une faible valeur fourragère ainsi que $D$. bursariifolia, toutes deux des zones arides, $D$. viscosa, de NouvelleZélande. D. thumbergiana, d'Afrique du Sud est de valeur moyenne. $D$. viscosa contient $10 \%$ de protéine; $1,8 \%$ de graisse; $64,7 \%$ d'hydrate de carbone

\section{Genre Heterodendron}

Ce genre comprend de petits arbres australiens dont les feuilles sont comestibles : Heterodendron diversifolium, $H$. oleifolium. Le dernier très répandu est considéré au Queensland comme un excellent arbre fourrager. Malheureusement il peut être cyanogénétique et causer des accidents mortels. La composition des feuilles est la suivante: protéine brute, 10,8; extrait éthéré, 3,5 ; cellulose brute, 19,5; extractif non azoté, 60,$1 ;$ cendres, 6,1 .

\section{Genre Pappea}

Pappea capensis, d'Afrique du Sud, est un bon arbre fourrager. Les baies auraient une bonne valeur nutritive. Après extraction de l'huile, les graines décortiquées donnent un produit qui renferme $38,3 \%$ de protéine, $4 \%$ de graisses et $43 \%$ d'hydrate de carbone.

\section{SAPOTACÉES \\ Genre Arganea}

Arganea sideroxylon localisé au Sud marocain, est un arbre dont les feuilles et les fruits sont mangés 
surtout par la chèvre et le chameau; les fruits sont conservés pour l'hiver et donnés aux vaches. La graine sert à la fabrication d'une huile comestible. Le tourteau est consomme par les chameaux et les vaches. Il renferme une saponine.

\section{Genre Sideroxylon}

Deux arbres d'Australie, de Nouvelle-Zélande, Sideroxylon pohlmannianum et $S$. novo-zclandicum sont considérés comme de bons fourrages en saison sèche.

\section{Genre Terminalia (V. Combretacées) Genre Ventilago}

Ventilago viminalis d'Australie, Nouvelle-Zélande, est considéré par les éleveurs du Queensland comme un des meilleurs arbres fourragers.

\section{Genre Wrightia}

Wrightia saligna du Queensland est un petit arbre qui est considéré comme excellent fourrage en saison sèche. $W$. tinctoria de l'Inde, est également mangé.

\section{SAXIFRAGACÉES}

\section{Genre Carpodetus}

Carpodetus serratus, petit arbre de NouvelleZélande, est mangé par le bétail.

\section{SCROFULARIACÉES \\ Genre Aptosimum}

Plusieurs plantes de ce genre forment en Afrique du Sud, des buissons bas dont les petites feuilles sont volontiers broutées : Aptosimum depressum, A. incisum, A. marlothii.

\section{Genre Hebe}

Ce genre, largement représenté en NouvelleZélande, comprend plusieurs espèces, dont le bétail mange les feuilles. La plus répandue est Hebe salicifolia, dont le feuillage est abondant.

\section{Genre Peliostomum}

En Afrique du Sud, deux espèces de ce genre sont recherchées : Peliostomum leucorrhizum caractéristique des terrains calcaires, et $P$. origanoïdes, à feuilles fines.

\section{Genre Scoparia}

Scoparia dulcis est une bonne plante fourragère, très répandue en Afrique Occidentale; elle est distribuée aux vaches comme galactogogue par les indigènes du Niger. Elle est utilisée comme fourrage en Amérique du Sud.

\section{Genre Selago}

Les espèces de ce genre, sud-africaines, sont indifférentes ou comestibles, Selago albida et S. speciosa sont bien appétées. $S$. minutissima est également mangée. Toutes ont de petites feuilles. Celles de $S$. speciosa ont la composition suivante : protéine brute, 7,9 ; extrait éthéré, 4,3 ; cellulose brute, 17,6; extractif non azoté, 62,8; cendres, 6,2.

\section{Genre Sutera}

Les espèces sud-africaines : Sutera crassicaulis, S. albiñora, S. atropurpurea sont mangées volontiers.

\section{Genre Walafreda}

Walefreda geniculata, très répandue en certaines régions d'Afrique du Sud est un bon fourrage. La composition est la suivante, en période de sécheresse : protéine brute, 12,4; extrait éthéré, 2,6; cellulose brute, 21,6; extractif non azoté, 56,1; cendres, 7,6 .

\section{SIMARUBECÉES \\ Genre Balanites}

Balanites zgyptiaca, arbre très répandu dans l'Afrique sahélienne, au Soudan égyptien, a des feuilles et pousses que mange surtout le chameau. Ces fruits peuvent être purgatifs.

\section{Genre Simarubacea}

Simarubacea sp. d'Afrique Orientale, très résistante à la sécheresse est excellente pour les chameaux du pays, mais serait dangereuse pour les chameaux importés.

\section{SOLANACÉES \\ Genre Lycium}

Des plantes buissonneuses de ce genre ont une valeur fourragère variable. Lycium arabicum (= intricatum) du Sahara, a des feuilles chamues aimées du chameau. En Asie Orientale, dans l'Inde, plusieurs especes sont de faible importance. En Afrique du Sud, sort mangées : $L$. afrum, $L$. arenicolum, L. horridum, L. hystrix, L. kraussii, L. tenue.

\section{Genre Solanum}

Solanum aviculare est un arbuste buissonneux de Nouvelle-Zélande dont le bétail mange les feuilles. Il est accusé d'être toxique, ainsi que $S$. sodomoum. En Australie, S. lasiophyllum, S. ellipticum sont également mangées.

Plusieurs espèces d'Amérique du Sud sont de bons fourrages : $S$. auriculatum (Brésil-Uruguay) dont les feuilles sont riches en protéines; S. bullatum, S. cerneum (Brésil), S. commersonil (Uruguạy), 
S. verbascifolium (Argentine); d'autres espèces sont suspectes: S. glaucum, S. elaœgnifolium, S. capsicastrum, S. sisymbrïfolium.

\section{Genre Whitania}

Whitania frutescens, d'Afrique du Nord, plus développée que les atriplex, est très résistante; les bovins et les moutons en sont friands; elle mériterait d'être répandue (Velu). W. somnifera est une

\section{Genre Guazuma}

Guazuma ulmifolia est un arbre de la savane vénézuélienne dont les feuilles sont mangées par Je bétail.

\section{Genre Hermannia}

A ce genre appartiennent de petits arbrisseaux d'Afrique du Sud, dont plusieurs espèces ont une certaine valeur fourragère; la plus répandue est

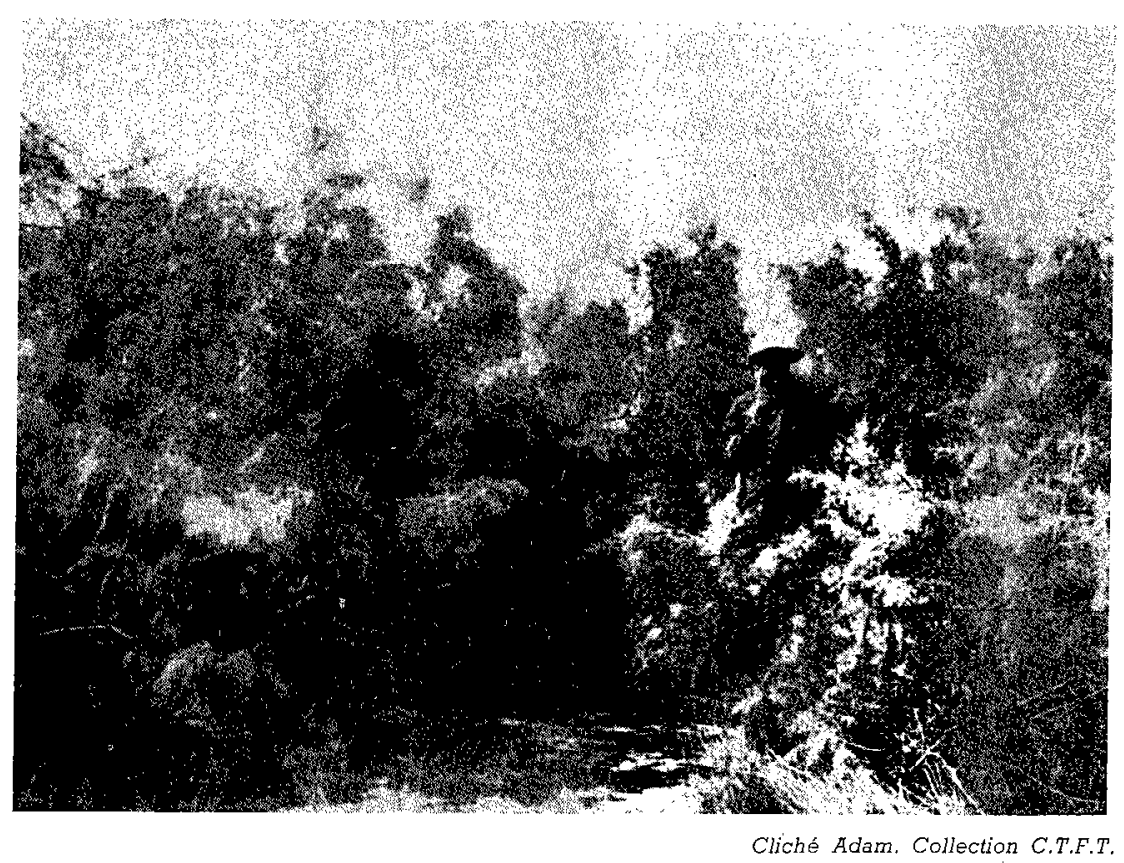

Fig. 4. - Tamarix senegalensis (A.O.F.):

espèce répandue en Asie, en Afrique, et qu'on signale comme toxique en Afrique du Sud (elle pourrait être photo-sensibilisante).

\section{STERCULIACÉS \\ Genre Brachychiton}

Plusieurs espèces australiennes de ce genre sont considérées comme étant parmi les meilleurs arbres fourragers. Brachychiton populneum (= Sterculia diversifolia) qui a été introduit en Ouganda, $B$. caudatum, $B$. rupestre, $B$, gregorii. Ce dernier est formé d'un tissu pulpeux, aussi le bétail mange-t-il feuilles et tronc de l'arbre abattu.

\section{Genre Dombeya}

Dombeya rotundifolia est un arbre du Transvaal dont les feuilles sont mangées.
Hermânnia linearifolia, à petites feuilles, qui permet à elle seule d'alimenter des moutons pendant assez longtemps; $H$. multiflora est également assez répandue comme la précédente dans les terrains de col line : $H$. spinosa, des régions sèches du Karoo est également mangée, malgré ses épines, Moins répandues sont ' $H$. condicans, ' $H$. linearis, $I I$. trifurcata, $H$. verticillata sont des espèces de zones sablonneuses, $H$. paucifolia purgative pourrait être dangereuse. $H$. linearifolia renferme $13,8 \%$ de protéine, $8,2 \%$ de graisse et $41,2 \%$ d'hydrate de carbone.

\section{Genre Sterculia}

Sterculia diversifolia ( $=S$. brachychiton populneum) est un arbre fourrager intéressant, d'Australie. C'est un arbre ornemental dont l'abattage est interdit en raison de sa valeur fourragère en saison sèche; il est très résistant à la sécheresse. En culture, 
il peut donner plusieurs tonnes de feuillage par acre ( 1 acre $=40$ ares env.). La composition est la suivante : eau, 35,61; cendres, 4,95; matières azotées, 10,35: cellulose, 14,54; hydrates de carbone, 32,81; matières grasses, 1,70.

S. rupestris, du Queensland, est également fourrager. De même $S$. cirenea, du Soudan égyptien, $S$. tomentosa, d'Afrique Occidentale, S. murex, du Transvaal dont les porcs mangent les graines. $S$. fœtida, d'Afrique Occidentale, a des graines qui après extraction de l'huile, donnent un produit qui renferme $31,7 \%$ de protéine, $7,2 \%$ de graisse et $43,5 \%$ d'hydrate de carbone.

\section{AMARICACÉES \\ Genre Myriceria}

Myriceria alopecurioïdes d'Asie centrale est un arbuste mangé par le chamean.

\section{Genre Tamaris}

De nombreuses espèces de ce genre sont répandues dans les déserts et sub-déserts. Ce sont en général des arbustes des terrains argileux et un peu saiés. Selon les espèces et la saison ils sont plus ou moins salés et amers et par suite, plus ou moins appréciés, c'est surtout le chameau qui les mange. On trouve en diverses régions d'Afrique : Tamaris articulata, T. africana, T. mannifera, T. macrocarpa, T. nilotica, T. pauciovulata, T. pentendra, en Arabie, Asie Mineure, des espèces africaines comme T. africana et d'autres comme T. aphylla; en Asie centrale T. Pallasii, dans l'Inde, T. gallica, T. orientalis; plusjeurs espèces existent aussi en Australie.

\section{TÉRÉBINTHACÉES \\ Genre Ailanthus}

Ailanthus excelsa est un arbre, parfois ornemental, de l'Inde, dont les feuilles peuvent être mangées.

\section{Genre Pistacia}

Pistacia atlantica est un arbre de la région circumméditerranéenne dont les feuilles sont très appréciées du chameau. Il en est de même pour $P$. integerrina et $P$. mutica, de l'Inde; $P$. terebinthus est aussi une espèce méditerranéenne qui est mangée.

\section{THYMÉLÉACÉES \\ Genre Thymelæa}

Thymelæa hirsuta, T. microphylla se rencontrent en Tripolitaine et sont surtout mangées par le chameau.

\section{TIIIACÉES \\ Genre Entelea}

Un arbuste de Nouvelle-Zélande, Entelea arborescens, a des feuilles très recherchées des bovins et des chevaux; à tel point qu'il tend à disparaître on cortaincs rógions.

\section{Genre Grewia}

Ce genre a de nombreux représentants qui sont des arbres fournissant un bon appoint fourrager. En Afrique du Sud : Grewia cana, G. flava, dont le betail mange les feuilles tombées en hiver; de même, G. hispida, G. occidentalis. En Afrique Orientale, $G$. platyclada, G. similis, G. bicolor. Cette dernière espèce se retrouve en Afrique Occidentale avec G. flavescens, G. mollis, G. villosa. Dans le Sahel et le Sahara, on rencontre G. betulifolia, G. populifolia dont les baies sucrées sont consommées; cette espèce existe aussi dans les régions semi-arides de l'Inde, avec G. pilosa, G. tiliæfolia.

\section{ULMACÉES \\ Genre Celtis}

Ce genre renferme un certain nombre d'espèces fourragères. Celtis sinensis est cultivé en de nombreux endroits du Queensland, comme arbre ornemental, et s'est répandu le long des rivières; on le considère comme un excel lent fourrage, de même que $C$. australis, des mêmes régions.

$C$. integrifolia, d'Afrique Orientale et Occidentale, est un fourrage de valeur; les indigenes mangent les feuilles comme légume ou les donnent aux animaux. C. kraussiana, C. rhamnifolia, d'Afrique du Sud ont des feuilles assez dures. $C$. occidentalis, $C$. pallida, C. reticulata sont des espèces américaines, dont les feuilles et les fruits sont mangés dans le Texas. C. spinosa, C. brasiliensis sont des arbres sud-anéricains. Au Brésil, les fruits de $C$. brasiliensis sont utilisés pour l'engraissement des animaux.

\section{Genre Trema}

Trema guineensis est un arbre d'Afrique Occidentale et d'Afrique Orientale dont les feuilles sont volontiers mangées par le bétail.

\section{URTICACÉES}

\section{Genre Soaresia}

Soaresia nitida est un arbre du Brésil, des régions semi-arides, dont les feuilles sont mangées volontiers.

\section{VERBENACEES}

Genre Avicennia

Le bétail, dans les zones littorales, mange parfois les feuilles des palétuviers. C'est ainsi que Avicennia 
marina var, resinifera, mangrove très répandu du Queensland est attaquée volontiers, on en coupe les branches pour les distribuer; le bétail mange aussi les fruits tombés mais ceux-ci, moisis, peuvent être dangereux. Sont aussi mangées quoique dures, les feuilles $d ' A$. officinalis de l'Inde, de NouvelleZélande, d'A. africana, des côtes atlantiques.

\section{Genre Lantana}

A ce genre appartiennent plusieurs espèces répandues en Asie; Afrique, Amérique, arbustes souvent utilisés comme haies, et qui ont marqué une grañde faculté d'adaptation au point qu'ils peuvent devenir envahissants. Les feuilles sont mangées en général par le bétail, surtout les moutons et les chèvres. Lantana camara est l'espèce la plus répandue. Les frutts pourraient être toxiques. Sont également mangées : L. brasiliensis, L. chamædrifolia, L. mista, L. radula, L. undulata, toutes du Brésil. $L$. crocea, $L$, sellowiana se seraient montrées toxiques en Australie, ainsi que des Lantana introduits aux îles Fidji oì on en a entrepris la destruction. Des Lantana peuvent causer des accidents de photosensibiliisation.

\section{Genre Lippia}

Lippia nodiflora, de l'Inde, est mangée par le chameau. Des espèces d'Afrique du Sud sont mangées par les moutons mais peuvent causer des acci-dents de photo-sensibilisation. $L$. trifida est une espèce des zones arides d'Argentine, $L$, addensis une espèce soudanaise.

\section{Genre Premna}

Premna resinosa, du Soudan égyptien, est une espèce très recherchée des chameaux.

\section{Genre Vitex}

A ce genre appartiennent des especes dont les feuilles sont consommées : Vitex cienkowskii d'Afrique Occidentale, $V$. lucens, de Nouvelle-Zélande qui pourrait être dangereux.

\section{VIOLACÉES \\ Genre Melicytus}

Un petit arbre de Nouvelle-Zélande, Melicytus ramiflorus est considéré comme l'espèce la plus intéressante à titre de fourrage complémentaire; les bovins surtout cn sonit friands ot lo fouillage est souvent distribué (arbre à vache). Les petites branches ingérées avec les feuilles pourraient causer des troubles digestifs et même la mort.

\section{ZYGOPHYLLACÉES \\ Genre Fagonia}

Fagonia arabica, F, fruticans, $F$. sinaica, du Sahara, $F$. cretica du Soudan égyptien, de Tripolitaine, ont des rameaux que mange le chameau.

\section{Genre Nitraria}

Dans le Sahara, le Soudan égyptien, la Tripolitaine, Nitraria tridentata ( $=N$. retusa) $N$. scholcrii, sont des plantes charnues, riches en salpêtre, que mange le chameau. $N$. schoberi en Nouvelle-Zélande a une faible valeur fourragère. La teneur en salpêtre de ces plantes peut causer des accidents chez le: chameau.

\section{Genre Tribulus}

Tribulus terrestris est une herbe répandue en de nombreuses régions chaudes du monde : région méditerranéenne, Afrique sahélienne, Afrique du Nord, Afrique du Sud, Indes. C'est un fourrage intéressant dans les zones sèches, à courte saison des pluies, en raison de sa rapidité de pousse; elle forme très vite un tapis parfois étendu dont les moutons en particulier, sont trè's friands. Elle cause des accidents de photosensibilisation.

Tr. alatus est également très répandu, Tr. macropterus, Tr. saharæ; tous deux du sud saharien, sont aussi de bons fourrages. Tr. maximus' est à la fois africain et américain.

\section{Genre Zygophyllum}

De nombreuses espèces du genre ont des feuilles charnues salées rappelant lès chénopodiacées. Zygophyllum album est un bon fourrage à chameau du sud saharien, à côté d'autres espèces du genre. $Z$. flexuosum ( $=Z$. retrofractum), $Z$. gilfilloni, $Z$. microcarpum, $Z$. morgsona, sont des espèces à feuilles succulentes, d'Áfrique du Sud, de valeur variable. Certaines espèces sud-africaines pourraient être dangereuses.

\section{FAMILLES DIVERSES \\ Genre Achyranthes}

Achyranthes aspera, d'Afrique Orientale, est mangée volontiers.

\section{Genre Aegle}

Aegle marmelos est un arbre de l'Inde dont les feuilles sont mangées.

\section{Genre Alphitonia}

Alphitonia excelsa est un arbre du Queensland qui est considéré comme excellent au point de vue fourrager; en saison sèche il serait moins recherché du bétail, probablement en raison de sa teneur en 
saponine. La composition des feuilles est la suivante : protéine ,13,9; graisse, 1,7; hydrate de carbone, 59,4,

\section{Genre Alstonia}

Un arbuste australien, Alstonia constricta dénommé "buisson quinine », en raison de la saveur amère de ses feuilles, est cependant mangé par les moutons; à une certaine époque, il peut être dangereux.

\section{Genre Borreria}

Des herbes de ce genre sont mangées par les animaux. Borreria radiata du Sahel africain, $B$. Ieiophylla d'Amérique du Sud.

\section{Genre Brosimum}

Brosimum galactodendron est un arbre à latex du Venezuela. Ce latex qui a une composition voisine du lait, est largement utilisé comme succédané du lait de vache dans l'alimentation humaine. B. alicastrum au Mexique, au Guatemala, a des feuilles comestibles et des fruits qu'on distribue aux animaux à l'engrais.

\section{Genre Buchanania}

Deux arbres de l'Inde, Buchanania angustifolia, $B$. lanzan, ont des feuilles comestibles.

\section{Genre Bursoninia}

Dans la savane ouverte vénézuelienne à Trachypogon, des arbres de ce genre dominent en certaines régions, sur les parties surélevées. Bursoninia verbascifolia est recherché du bétail, qui préfère ses feuilles aux herbes, même quand Il s'agit de repousses après le feu.

\section{Genre Butea}

Butea frondosa est un arbuste de l'Inde dont les feuilles sont mangées.

\section{Genre Cedrela}

Cedrela toona, arbre de l'Inde, a des feuilles comestibles.

\section{Genre Cercidium}

Les pousses et les fruits de Cercidium peninsulare (Californie, Mexique) sont mangées par le bétail et provoquent un engraissement rapide.

\section{Genre Condalla}

Des buissons dont les feuilles sont mangées par le bétail, sont constitués par des espèces américaines du genre Condalia microphylla, de la pampa argentine, $C$. lineata de la sierra argentine et chilienne, C. obtusifolia du Texas.

\section{Genre Crescentia}

Un arbre du Salvador, Crescentia alata, a des fruits que mangent les animaux quand ils sont tombés à terre, verts ou en cours de fermentation, en saison sèche; en certaines régions, ils constituent à ce moment la seule source d'aliments. Il's ont la réputation de donner un fromage de bonne qualité.

\section{Genre Disperma}

Les arbustes de ce genre et particulièrement Disperma trachyphyllum, de l'Est africain, tiennent une place importante en zone désertique; cet arbuste

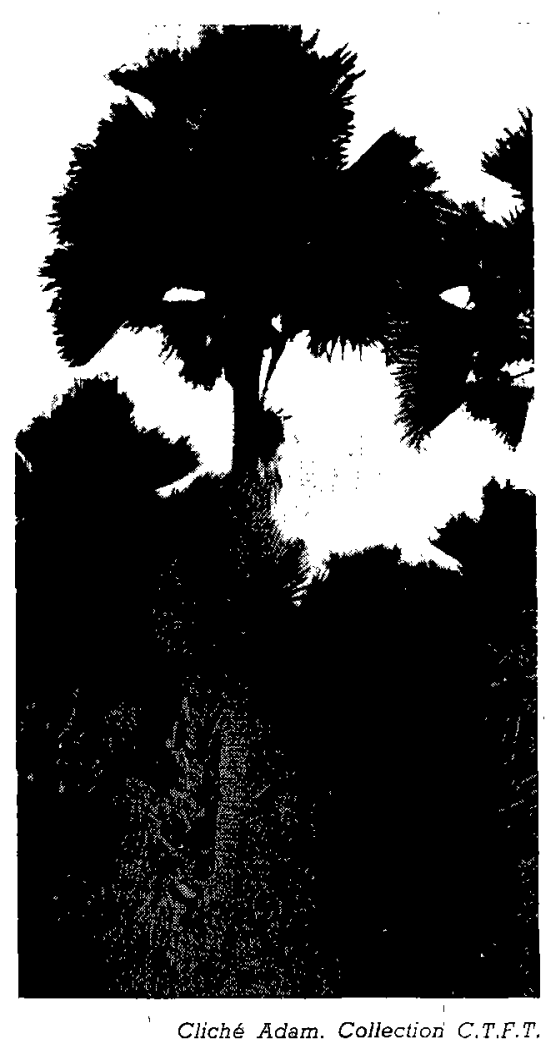

Fig. 5. - Borassus flabellifer (A.O.F.).

occupe de grands espaces en raison de sa résistance au feu et à la sécheresse. Les feuilles bien que peu digestibles, ont une bonne composition (protéines 22,8; graisses, 2,7; H. de C., 43,2; cellulose, 12,8), D. parviflorum, D. quadrisepalum, également do l'Est africain, ont des propriétés analogues.

\section{Genxe Dolichandrone}

Un arbre de l'Inde, Dolichandrone falcata, a des feuilles comestibles, un autre arbre du Queensland, $D$. heterophylla, est un bon fourrage. 


\section{Genre Eichhornia'}

Eichhornia azurea est considérée comme une des meilleures herbes fourragères de certaines régions du Brésil. E. natans est une bonne espèce, peut répandue, de l'Afrique Occidentale, E. crassipes, véritable fléau par son envahissement des cours d'eau en Indochine, peut être utilisé, coupé au couperacines, aspergé d'eau et fermenté.

\section{Genre Gmelina}

Les feuilles de Gmalina arborea sont appréciées dans diverses régions de l'Inde.

\section{Genre Hardwickia}

Hardwickia binata, arbre de diverses provinces de l'Inde, joue un rôle fourrager important; son élagage est réglementé surtout là où, l'élevage à

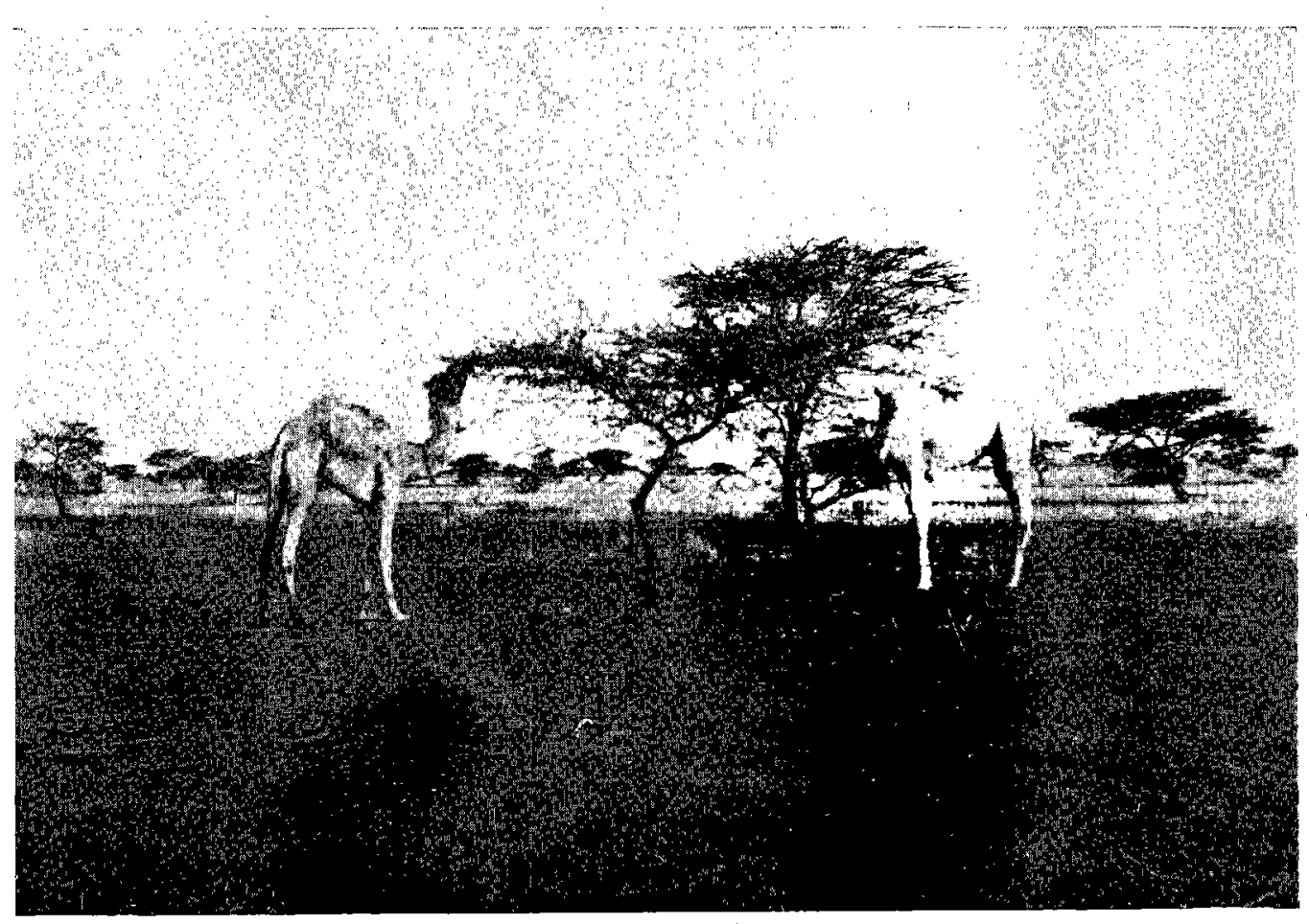

Fig. 6. - Pâturage arbustif type.

Photo Cocheteux

\section{Genre Eriolœna}

Eriolœna hookeriana est un arbre fourrager de l'Inde.

\section{Genre Eugenia}

Les feuilles d'Eugenia jambolana, arbre de l'Inde, sont considérées comme un bon fourrage.

\section{Genre Evodia}

Un arbre du Bengale, Evodia fraxinifolia a des feuilles comestibles. On le cultive autour de certains villages pour distribuer les branches au bétail.

\section{Genre Feronia}

Le bétail mange les feuilles de Feronia elephantum, arbre de l'Inde.

\section{Genre Gliciridia}

A Ceylan, Gliciridia maculata a des feuilles comestibles.
I'étable étant la règle, on y a recours abondamment, notamment pour les vaches laitières; on fait parfois provision des feuilles pour la saison sèche.

\section{Genre Helicteres}

Dans l'Inde (Bombay) on distribue les feuilles d'Helicteres isora au bétail.

\section{Genre Kydia}

Kydia calycina de l'Inde, a des feuilles comestibles.

\section{Genre Mikania}

Mikania micrantha est une herbe perenne d'Uruguay, qui est bien recherchée du bétail, assez pour qu'elle tende à disparaître. A Ceylan, $M$. scandens, plante grimpante introduite, a des feuilles et des pousses succulentes très rocherchées; elle a envahi les cultures dans les régions humides. 


\section{Genre Nothophagus}

Un arbre de ce genre, Nothophagus dombeyi, du Chili, offre cette particularité que le bois décomposé des troncs tombés est mangé avidement par le bétail, de préférence aux graminées.

\section{Genre Pongamia}

Le bétail mange les feuilles de Pongamia glabra de l'Inde.

\section{Genre Scævola}

Scævola spinescens, très répandu en Australie de l'Ouest, est important à la fois par ses feuilles et

Analyses d'arbres, arbustes et buissons d'Afrique Occidentale

\begin{tabular}{|c|c|c|c|c|c|c|c|}
\hline ESPËCES & PARTEE ANALYSÉE & EENU & $\begin{array}{c}\text { PROTELINE } \\
\text { brute }\end{array}$ & $\begin{array}{c}\text { EXTRAIT } \\
\text { éthéré }\end{array}$ & $\begin{array}{l}\text { CBLLULOSB } \\
\text { brute }\end{array}$ & $\mid \begin{array}{c}\text { EXTRAIT } \\
\text { non azoté }\end{array}$ & CENDRES \\
\hline Acacia arabica .... & Pousses et feuilles & $»$ & 12,9 & 12,6 & 11,3 & 56,7 & 6,4 \\
\hline Acacia albida....... & Gousses & 10,6 & 9,7 & 1,1 & 23,4 & 51,96 & 3,24 \\
\hline Adansonia digitata. & Graines & 12,1 & 12,7 & 13,2 & 25,6 & $44 ; 5$ & 4 \\
\hline Afzelia africana .... & Feuilles & $》$ & 12,2 & 3,5 & 40,2 & 38,4 & 5,8 \\
\hline Anona senegalensis & id. & $»$ & 9,2 & $»$ & $»$ & $"$ & 7,7 \\
\hline Balanites ægyptiaca & id. & $\eta$ & 11,6 & 4.2 & 13,6 & 57,9 & 12,7 \\
\hline Bauhinia reticulata... & id. & ») & 19,2 & 22,7 & 11,1 & 44,3 & 2,7 \\
\hline Bauhinia rufescens .... & Fruits & 9,2 & 11,1 & 1,5 & 33,6 & $49 ; 3$ & 4,4 \\
\hline Bauhinia thonningii...... & Gousses & 4.7 & 6,8 & 2,4 & 23,7 & 63,8 & 4,9 \\
\hline Boscia sp. .......... & Feuilles & $»$ & 20,2 & 1,5 & 31,8 & 40,3 & 6,2 \\
\hline Combretum sp. ....... & id. & $\gg$ & 6,2 & $»$ & $»$ & 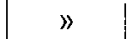 & 4,4 \\
\hline Dalbergia sp. . . . . . & id. & 》) & 19,8 & 3,3 & 14,1 & 45,5 & 7,9 \\
\hline Diospyros mespiliformis & id. & ») & 6,5 & 12,1 & 14,1 & 59,4 & 7,0 \\
\hline Guiera senegalensis ... & id. & $\gg$ & 9,6 & 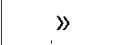 & $»$ & i) & 5,2 \\
\hline Khaya senegalensis..... & id. & $\$ 3$ & 21.7 & 4,3 & 14,8 & $52 ; 2$ & 6,9 \\
\hline Parkia lilicoïdea .... & Graines & $»$ & 17,2 & 8,2 & 12,2 & 57,6 & 4,9 \\
\hline Prosopis oblonga. . & Pousses et feuilles & 7,9 & 10,2 & 6,8 & 22,9 & 49,46 & 2,74 \\
\hline Pterocarpus erinaceus .... & Feuilles & $»$ & 19 & 2,7 & 35,5 & 35,6 & 7,2 \\
\hline Stereospermum kunthianum. & id. & 3) & 4,1 & $»$ & $\gg$ & $»$ & 20,3 \\
\hline Vitex cienkowskii ........ & id. & 3 & 10,4 & 2,8 & 35,2 & 44,5 & 7,1 \\
\hline Zizyphus jujuba..... & id. & $》$ & 11,8 & 4,3 & 14,3 & 61 & 8,6 \\
\hline
\end{tabular}

Analyses d'arbres, arbustes et buissons d'Afrique Orientale

\begin{tabular}{|c|c|c|c|c|c|c|c|}
\hline ESPECES & PARTIE ANALYSEEE & EAJ & $\begin{array}{l}\text { PROTEINE } \\
\text { brute }\end{array}$ & $\begin{array}{l}\text { EXTRAIT } \\
\text { éthéré }\end{array}$ & $\begin{array}{l}\text { CFLLILLSBSB } \\
\text { brute }\end{array}$ & $\mid \begin{array}{c}\text { EXTRAIT } \\
\text { non azoté }\end{array}$ & CENDRES \\
\hline Acacia albida.. & Gousses & $»$ & 8,82 & 1,44 & 24,40 & 61,74 & 3,6 \\
\hline Acacia spirocarpa & id. & 10 & 12,3 & 1,8 & 22,4 & 57,9 & 5,6 \\
\hline Adansonia digitata. & Fruit (pulpe+grains) & 11 & 11,1 & 6,6 & 24,6 & 52,9 & 4,7 \\
\hline Dichrostachys glomerata ... & Gousses & $»$ & 11,4 & 1,2 & 25,6 & 56,4 & 5,5 \\
\hline Disperma trachyphyllum & Jeunes feuilles & 72 & 13 & 2,7 & 13,4 & 52,6 & 18,3 \\
\hline Ficus sycomorus ...... & Feuilles & 70 & 13,5 & 2,1 & 14,3 & 54,7 & 15,4 \\
\hline Phytelepas macrocarpa.... & Fruit & 11,39 & 4,63 & 0,92 & 6,89 & 75,09 & 1,8 \\
\hline Eriodendron anfractuosum... & Graines & 13,6 & 27 & 7,1 & 25,8 & 22 & 6,3 \\
\hline
\end{tabular}


ses fruits. Ces derniers sont mangés aussi par l'homme.

\section{Genre Sechium}

Sechium edule, d'Amérique du Sud (Brésil), du
Mexique, de l'Inde, a des feuilles qu'on utilise fragmentées ou ensilées. Les fruits, les racines sont également utilisables. En Indochine, on distribue les fruits aux bœufs. Ces fruits renferment 11 pour 1000 de matières albuminoïdes et 1 pour 1000 de matières grasses (Rémoná et Winter 1944).

\section{Analyse d'arbres, axbustes et buissons du Soudan égyptien}

\begin{tabular}{|c|c|c|c|c|c|c|c|}
\hline ESPF.CES & PARTIE ANALYSÉE & EAU & $\begin{array}{c}\text { PROTÉINE } \\
\text { brute }\end{array}$ & $\begin{array}{c}\text { EXTRAIT } \\
\text { éthéré }\end{array}$ & $\begin{array}{l}\text { CELLILOSE } \\
\text { brute }\end{array}$ & $\begin{array}{c}\text { EXTRAIT } \\
\text { non azoté }\end{array}$ & CENDRES \\
\hline Acacia albida.............. & Folioles & 63,7 & 17,1 & 2,3 & 12,1 & 59,7 & 8,4 \\
\hline Acacia ehrenbergiana ..... & Feuilles & 57,2 & 22,5 & 2,3 & 20,1 & 50,1 & 5 \\
\hline Acacia mellifera........... & id. & 65.9 & 21,3 & 2,6 & 14,1 & 53.4 & 8.6 \\
\hline Acacia seval. . & Gousses vertes & 68,5 & 21,3 & 1,8 & 18,6 & 52,2 & 6,1 \\
\hline Acacia seyal ..... & Gousses mûres sèches & 4,7 & 20,9 & 1,9 & 20,2 & $47 ; 6$ & 9,3 \\
\hline Capparis spinosa... & Feuilles & 69,6 & 13,8 & 1,5 & 7,9 & 53,7 & 23,2 \\
\hline Maerua crassifolia... & id. & 10,1 & 15,5 & 1,3 & 6,1 & 53,3 & 23,8 \\
\hline Prosopis chilensis .... & Gousses & 5,6 & 11,5 & 2,2 & 26,9 & 54 & 5,4 \\
\hline Prosopis juliflora ...... & Feuilles & 58,2 & 19 & 2,9 & 21,6 & 47,9 & 8,5 \\
\hline Salvadora persica......... & id. & 69,6 & 13,8 & 1,5 & 7,9 & 53,7 & 23,1 \\
\hline Zizyphus spina christi ..... & id. & 48,3 & 18,3 & 1,8 & 6,8 & 64,7 & 8,5 \\
\hline
\end{tabular}

\section{Analyses d'arbres, arbustes et buissons de Rhodésie}

\begin{tabular}{|c|c|c|c|c|c|c|c|}
\hline - ESPËCES & PARTIE ANALYSEEE & EAU & $\left\{\begin{array}{c}\text { PROTÉINE } \\
\text { brute }\end{array}\right.$ & $\begin{array}{l}\text { EXTRAIT } \\
\text { éthéré }\end{array}$ & $\begin{array}{l}\text { CElLULOSE } \\
\text { brute }\end{array}$ & $\begin{array}{l}\text { EXTRAIT } \\
\text { non azoté }\end{array}$ & CENDRES \\
\hline Agave americana . . & Feuilles sèches & 15,1 & 6,1 & 1,3 & 15,6 & 64,5 & 12,4 \\
\hline Albizzia amara $\ldots \ldots \ldots \ldots$ & Gousses & 9,3 & 13,5 & 7,6 & 36 & 39,2 & 3,6 \\
\hline Ceratonia siliqua $\ldots . . \ldots \ldots$ & Graines & $\gg$ & 18,2 & 2,8 & 8,6 & 66,7 & 3,6 \\
\hline Cussonia spicata & Feuilles sèches & 22,1 & 8,6 & 3,5 & 19,6 & 60,3 & 7,8 \\
\hline Dichrostachys glomerata & Graines & $\gg$ & 20 & 2,2 & 21,8 & 51,1 & 4,9 \\
\hline Kigelia pinnata $\ldots \ldots \ldots \ldots$ & Fruits & 85,4 & 24,8 & 2,5 & 11,1 & 33,5 & $\gg$ \\
\hline Mimosa sp.............. & Farine & 7,1 & 12,1 & 1 & 22,8 & 59,6 & 4,5 \\
\hline Passiflora quadrangularis .. & Feuilles & 8,9 & 16,9 & 5,1 & 11,0 & 57,2 & 8,0 \\
\hline Phytolacca octandra ....... & Foin & $》$ & 26,5 & 2,4 & 17,4 & 40,5 & 13,1 \\
\hline Portulaceria afra ... & id. & $»$ & 8.5 & 3,8 & 20,9 & 50,7 & 9.8 \\
\hline Salix babylonica.......... & Feuilles sèches & 13,9 & 16,7 & 2,9 & 18 & 52,1 & 10,2 \\
\hline Swartzia madagascariensis . & Gousses & $\gg$ & 6,2 & 1,2 & 23,2 & 66,7 & 2,7 \\
\hline Viscum verrucosum........ & Feuilles sèches & $»$ & 13,6 & 5,2 & 22,4 & 49,2 & 8,5 \\
\hline
\end{tabular}


Analyses des feuilles de quelques arbres et arbustes d'Afrique du Sud

(Henrici)

\begin{tabular}{|c|c|c|c|c|c|c|c|c|}
\hline ESPËCES & GENDRES & $\begin{array}{l}\text { PRotínL } \\
\text { brute }\end{array}$ & $\begin{array}{l}\text { DXTRtII } \\
\text { éthéré }\end{array}$ & $\begin{array}{l}\text { Cillibis } \\
\text { brute }\end{array}$ & $\begin{array}{l}\text { ExlRull } \\
\text { non } \\
\text { azoté }\end{array}$ & $\mathrm{P} 2 \mathrm{O5}$ & $\mathrm{CaO}$ & PARTIE' ANALYSÉE \\
\hline Acacia detinens.... & 10,62 & 19,82 & 6,70 & 23,17 & 39,69 & 0,38 & 4,61 & Feuilles vertes \\
\hline Acacia litakunensis... & 8,72 & 19,20 & 6,10 & 11,56 & 54,37 & 0,39 & 3,18 & id. \\
\hline Cadaba juncea . & 6,40 & 19,25 & 1,82 & 14,38 & 58,15 & 0,35 & 0,56 & $\begin{array}{c}\text { Feuilles et branches } \\
\text { vertes }\end{array}$ \\
\hline Capparis albitrunca. & 10,01 & 19,91 & 4,52 & 20.04 & 45,51 & 0,29 & 1,81 & Feuilles vertes \\
\hline Caroxylon (= Salsola) sp.... & 17,65 & 23,45 & 2,26 & 10,03 & 46,61 & 0,50 & 3,18 & $\begin{array}{c}\text { Feuilles et pousses } \\
\text { vertes }\end{array}$ \\
\hline Rhigozum trichotomum .. & 7,02 & 14,44 & 12,79 & 15,60 & 50,15 & 0,24 & $»$ & Feuilles vertes \\
\hline Lycium sp. . . . . . . . & 13,97 & 27,21 & 2,68 & 14,60 & 41,56 & 1,25 & 3,67 & id. \\
\hline Combretum apiculatum... & $\gg$ & 4,29 & $»$ & $》$ & 》 & 0,11 & $»$ & Feuilles tombées \\
\hline Boscia albitrunca...... & $»$ & 15,31 & $»$ & $\gg$ & $»$ & 0,22 & $»$ & Feuilles vertes \\
\hline Grewia flava . . & $》$ & 10,15 & $»$ & $»$ & $》$ & 0,22 & $\gg$ & id. \\
\hline Copaïfera mopane....... & $》$ & 10,76 & 》 & $\gg$ & $»$ & 0,22 & 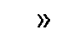 & id. \\
\hline
\end{tabular}

\section{Composition de plantes buissonnenses du Karoo (Afrique du Sud)}

(Henrici)

\begin{tabular}{|c|c|c|c|c|c|}
\hline ESPECES , & $\begin{array}{l}\text { MATIERE } \\
\text { organique }\end{array}$ & $\begin{array}{l}\text { PROTÉINL } \\
\text { Drute }\end{array}$ & $\begin{array}{c}\text { EXTRAIT } \\
\text { éthéré }\end{array}$ & $\begin{array}{l}\text { CELLULOSE } \\
\text { brute }\end{array}$ & $\begin{array}{l}\text { EXTRAIT } \\
\text { non azoté }\end{array}$ \\
\hline Olea verrucosa $\ldots \ldots \ldots \ldots \ldots \ldots \ldots \ldots \ldots$ & 67,4 & 33,7 & 57,4 & 35,6 & 85 \\
\hline 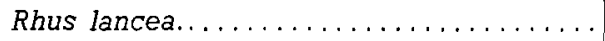 & 49,44 & 36,3 & 82,5 & 0 & 70,8 \\
\hline Salsola glabrescens .............. & 70,5 & 69,8 & 97,5 & 67,6 & 78,4 \\
\hline Atriplex capensis (excellent) .. & 91 & 81,7 & 92,4 & 100 & 92,3 \\
\hline id. $\quad$ (pauvre) $\ldots$. & 42,4 & 72,7 & 33 & » & 47,7 \\
\hline id. $\quad(\sec ) \ldots \ldots \ldots$ & 68,7 & 88,7 & 59,6 & 56,8 & 70,3 \\
\hline Lotononis divaricata.......... & 48,1 & 47,1 & 50,6 & 42,2 & 54,5 \\
\hline Sutherlandia microphylla...... & 51,5 & 84,3 & 53,9 & 0,8 & 74,5 \\
\hline Walafrida geniculata ....... & 67,3 & 55,6 & 57,4 & 55,8 & 76,8 \\
\hline Pentzia incana................. & 67,3 & 75 & 74,1 & 58,4 & 72,5 \\
\hline Phymaspermum parvitolium ....... & 47,9 & 56,1 & 55,6 & 39,7 & 57,8 \\
\hline Tripteris pachypteris................. & 51,5 & 66,8 & 11,8 & 16,1 & 77,1 \\
\hline
\end{tabular}


Plantes buissonneuses d'Asie Orientale et Centrale

\begin{tabular}{|c|c|c|c|c|c|c|}
\hline ESPECES & PÁRTIE RNALYSÉE & $\begin{array}{c}\text { PROTÉLNE } \\
\text { brute }\end{array}$ & $\begin{array}{c}\text { EXTRAIT } \\
\text { éthéré }\end{array}$ & GLLLELSR & $\begin{array}{c}\text { EXTRAIT } \\
\text { non azoté }\end{array}$ & CENDRES \\
\hline Agriophyllum arenarium & Feuilles & 6,2 & 2,3 & 25 & 55,6 & 11 \\
\hline Alhagi camelorum ...... & Plantes sèches & 5,1 & 1,8 & 45,9 & 40,8 & 6,4 \\
\hline Anabasis aphylla....... & id. & 7,1 & 3,2 & 25,6 & 15,9 & 18,2 \\
\hline Artemisia arenaria ...... & Plante jeune & 14,9 & 2,9 & 16,4 & 56,3 & 9,5 \\
\hline Artemisia herba alba .......... & Plante en boutons & 6,3 & 4,3 & 43,5 & 39,9 & 6 \\
\hline Artemisia maritima... . . . & Fin de végétation & 5 & 3,7 & 47,5 & 37,3 & 6,5 \\
\hline Artemisia persica ....... & Plante en fleurs & 11,3 & $=4,2$ & 33,6 & 42,6 & 8,3 \\
\hline Atriplex cana................. & Plante entière & 7,7 & 1,7 & 38,2 & 38,7 & $\gg$ \\
\hline Atriplex pedunculata .... & id. & 5,5 & 3,2 & 32,9 & 28,6 & 28,7 \\
\hline Ceratocarpus arenarius .. & Feuilles & 9,8 & 1,6 & 21 & 57 & 10,6 \\
\hline Ephedra strobiculata $\therefore$. & Plante en fleurs & 16,5 & 3 & 30,4 & 35,3 & 14,8 \\
\hline Gamanthus ovinus ..... & Plante flétrie & 7,2 & 1,8 & 9,7 & 44,7 & 36,9 \\
\hline Malva sp. . . . . . . . & Plante en fleurs & 11,2 & 4,5 & 20,8 & 40,9 & 14,5 \\
\hline Petrosimonia brachiata ... & id. & 6 & 2,2 & 48,8 & 26,6 & 16,4 \\
\hline Salsola arbuscula & id. & 8,5 & 3 & 30,3 & 43,5 & 14,7 \\
\hline Salsola kali ....... & Plante en graines & 13,1 & 2,6 & 22 & 48,6 & 13,7 \\
\hline Suaeda maritima ... & Plante portant fruits & 6,6 & 2,8 & 19,3 & 59,2 & 12,1 \\
\hline
\end{tabular}

Arbres et arbustes de 1'Inde

\begin{tabular}{|c|c|c|c|c|c|c|c|}
\hline ESPECES & PARTIE ANALYSÉE & EAU & $\begin{array}{c}\text { PROTÉINE } \\
\text { brute }\end{array}$ & $\begin{array}{l}\text { EXTRAIT } \\
\text { éthéré }\end{array}$ & $\begin{array}{c}\text { CELLLLOSY } \\
\text { brute }\end{array}$ & $\begin{array}{c}\text { EXTRAIT } \\
\text { non azoté }\end{array}$ & CENDRES \\
\hline Adinia cordifolia & Feuilles & $\eta$ & 15,3 & 3,9 & 12,7 & 60,2 & 7,9 \\
\hline Azadirachta indica... & id. & 64 & 15 & 3 & 13,8 & 57 & 10,6 \\
\hline Bauhinia variegata......... & id. & $》$ & 13,2 & 2,1 & 29,4 & 46,8 & $8,5^{\prime}$ \\
\hline Dalbergia sissoo . . . . . . . . & id. & 74,9 & 24,1 & 2 & 12,5 & 54,8 & 6,6 \\
\hline Eugenia jambolana.... & id. & 62,9 & 7,9 & 2,6 & 20,7 & 61,7 & 7 \\
\hline Ficus infectoria........... & id. & 58,1 & 11,7 & 2,7 & 25,5 & 51,8 & 8,3 \\
\hline Ficus religiosa... . & id. & 85,4 & 22,6 & 2,8 & 12,2 & 46 & 16,4 \\
\hline Grewia oppositifolia ....... & id. & $》$ & 16,4 & 8,4 & 16,6 & 43,8 & 14,9 \\
\hline Mangifera indica....... & id. & 56 & 8,1 & 2,7 & 28 & 50,6 & 10 \\
\hline Moringa oleifora .... & id. & 75 & 26,6 & 6,9 & 3,5 & 54 & 9,1 \\
\hline Opuntia sp. .......... & Fruits & 85,1 & 4 & 2,9 & 12,5 & 58,1 & 23,6 \\
\hline Pithecolobium dulce ... & id. & 80,8 & 13,4 & 1,6 & \multicolumn{2}{|c|}{82,9} & 2,1 \\
\hline Sesbania ægyptiàca & Feuilles & 68,2 & 17,8 & 4,2 & 28 & 39,2 & 8,9 \\
\hline Tamarindus indica. . & id. & 70,2 & 14,7 & 3,7 & 24,4 & 50,3 & 6,9 \\
\hline Zizyphus jujuba........... & id. & 58,7 & 14 & 2,7 & 14,9 & 58,5 & 10,3 \\
\hline
\end{tabular}


Arbres, buissons et arbustes d'Australie de 1'Ouest

(Meadly)

\begin{tabular}{|c|c|c|c|c|c|c|c|}
\hline ESPECEES & $\begin{array}{l}\text { VALEUR } \\
\text { fourragere }\end{array}$ & $\begin{array}{l}\text { MATÉRIEL } \\
\text { analysé }\end{array}$ & $\begin{array}{c}\text { Mutiklel } \\
\text { organique }\end{array}$ & $\begin{array}{c}\text { PROTÉINE } \\
\text { brufe }\end{array}$ & $\begin{array}{l}\text { EXTRAIT } \\
\text { éthéré }\end{array}$ & CELLLIIEL & $\begin{array}{l}\text { EXIRAT? } \\
\text { non azote }\end{array}$ \\
\hline Acacia linophylla ....... & Gousses bonnes & Gousses & 95,4 & 12,8 & 5 & 28,2 & 49,4 \\
\hline Acacia tetragonophylla & Phyllodes et gousses & Phyllodes & 94,7 & 16,6 & 2,5 & 32,7 & 42,9 \\
\hline Atriplex hymenotheca .. & Feuilles bonnes & Feuilles & 73,7 & 11,4 & 2 & 14,2 & 46,1 \\
\hline Atriplex rhagodioïdes .. & id. & id. & 89,1 & 10 & 6 & 13,2 & 60 \\
\hline Atriplex semibaccata & id. & id. & $》$ & 18,8 & 2,4 & 18,7 & 39,1 \\
\hline Rochia brevifolia & id. & id. & » & 24,8 & 2,5 & $1: 1,1$ & 33,5 \\
\hline Rochia pyramidata & id. & $\begin{array}{l}\text { Jeunes } \\
\text { pousses }\end{array}$ & 69,2 & 22 & 2,1 & 10,3 & 34,8 \\
\hline Rochia sedifolia......... & id. & Feuilles & 83,2 & 17,2 & 2,6 & 22 & 41,5 \\
\hline Scaevola spinescens .... & Feuilles et fruits & id. & 90,9 & 12,5 & 5,4 & 10,7 & 62,3 \\
\hline Solanum ellipticum ..... & Feuilles & id. & 86 & 15,2 & 5,2 & 23,5 & $44, \breve{1}$ \\
\hline Trichinium exaltatum ... & Feuilles bonnes & id. & 91,9 & 15,7 & 2,1 & 22,5 & 52,5 \\
\hline
\end{tabular}

\section{Zxbres fourragers d'Rnstralie}

(Breakwell 1923)

\begin{tabular}{|c|c|c|c|c|c|c|c|c|}
\hline ESPECES & EAU & CENDRES & $\begin{array}{l}\text { MAT. } \\
\text { AlvurI. }\end{array}$ & CELLtiis. & H. de C. & GRAISSES & $\begin{array}{c}\text { pitullat } \\
\text { nutritive }\end{array}$ & $\begin{array}{l}\text { VALEUR } \\
\text { nulritive }\end{array}$ \\
\hline Acacia aneura $\ldots \ldots \ldots \ldots$ & 39,06 & 3,60 & 9,06 & 29,90 & 15,83 & 2,55 & 12,5 & 30,75 \\
\hline Casuarina lepidophloia .. & 11,70 & 5,66 & 9,06 & 46,86 & 23,92 & 2,80 & $1,3,25$ & 39,25 \\
\hline Scaevola spinescens .......... & 19,79 & 13,22 & 9,94 & 44,76 & 10,72 & 1,57 & $1: 5,7$ & 24,25 \\
\hline Eremophila longifolia . & 51,59 & 3,70 & 8,87 & 5,43 & 29,66 & 0,75 & $1,3,5$ & 40,25 \\
\hline Brachychiton populneum & 35,61 & 4,99 & 10,35 & 14,54 & 32,81 & 1,70 & $1 / 3,5$ & 46 \\
\hline Acacia pendula ....... & 48,45 & 3,60 & 9,06 & 29,90 & 15,83 & 2,55 & $1 / 2,5$ & 30,75 \\
\hline Heterodendron oleifolium & 12,27 & 4,84 & 15,75 & 16,36 & 48,58 & 2,20 & $1: 3,5$ & 69 \\
\hline Atalaga hemiglauca...... & 35,87 & 6,27 & 14,62 & 19,50 & 22,55 & 1,19 & $1 / 2$ & 39,75 \\
\hline
\end{tabular}


Arbres, arbustes et buissons des iles Hawai et de la Jamaïque

\begin{tabular}{|c|c|c|c|c|c|c|}
\hline ESPËCES & PARTIE ANALYSÉE & $\begin{array}{c}\text { PROTELNE } \\
\text { brute }\end{array}$ & $\begin{array}{l}\text { EXTRNIT } \\
\text { éthéré }\end{array}$ & $\begin{array}{l}\text { chlullosh } \\
\text { brute }\end{array}$ & $\begin{array}{l}\text { EXTRAIT } \\
\text { non azoté }\end{array}$ & CENDRES \\
\hline Bambusa vulgaris (Jamaïque) & Feuilles & 19 & 0,5 & 28,8 & 34,8 & 16,9 \\
\hline Brosimum alicastrum (Hawaï). . . . & Plante verte & 13,9 & 3,4 & 26,5 & 48,3 & 7,9 \\
\hline Cibotium chamissoi (Hawaï)..... & Tronc (fougère) & 3,7 & $\gg$ & $»$ & 82,1 & $»$ \\
\hline Cordyline terminalis (Hawai).... & Feuilles & 13,9 & 3,1 & 30,9 & 43,1 & $9 ; \mathrm{I}$ \\
\hline Desmanthus virgatus (IIawaï).... & Plantes vertes & 11,5 & 2,1 & 43,4 & 37,2 & 5,8 \\
\hline Leucoena glauca (Hawaï) ....... & Cimes vertes & 18,8 & 2,3 & 37,7 & 34,5 & 6,6 \\
\hline Melicocca bijuga (Jamaïque).... & Graines & 11,6 & 1,2 & $I^{\prime} 1,2$ & 73,4 & 2,7 \\
\hline Opuntia sp. (Hawai) .......... & Feuilles & 7,7 & 1,5 & 10,5 & 59,4 & 21 \\
\hline Pithecolobium saman (Jamaïque). & Gousses & 12,8 & 0,7 & 14,5 & 69,6 & 2,4 \\
\hline Prosopis chilensis (Hawaï)....... & $\begin{array}{c}\text { Farine de graine } \\
\text { (sèche) }\end{array}$ & 9,5 & 1,5 & 23,2 & 57,9 & 4,3 \\
\hline Trophis americana (Jamaique) .. & $\begin{array}{c}\text { Feuilles et pousses } \\
\text { (sèches) }\end{array}$ & 11,2 & 5,9 & 26,5 & 48,4 & 8 \\
\hline
\end{tabular}

\section{Arbres, arbustes et buissons d'Ạnérique du Nord}

(Texas)

\begin{tabular}{|c|c|c|c|c|c|c|}
\hline ESPËCES & PARTIE ANALYSÉE & $\begin{array}{c}\text { PROTEINE } \\
\text { brute }\end{array}$ & $\begin{array}{l}\text { EXTRAIT } \\
\text { éthéré }\end{array}$ & $\begin{array}{l}\text { CFLLILIOSE } \\
\text { brute }\end{array}$ & $\begin{array}{c}\text { EXTRAIT } \\
\text { non azoté }\end{array}$ & CENDRES \\
\hline Acacia roemeriana ............ & Feuilles & 22,6 & 4 & 8,4 & 56,1 & 9 \\
\hline Atriplex lentiformis . . & Plante entière & 8,3 & 2,1 & 28,8 & 55,7 & 5 \\
\hline Berberis trifoliata............. & Feuilles & 11 & 2,5 & 32,6 & 50,7 & $3 ; 2$ \\
\hline Boerhavia tenuifolia ............. & Plante entière & 11,4 & 2,2 & 24,3 & 52,6 & 9,5 \\
\hline Diospyros texana............ & Feuilles & 12,3 & 10,3 & 20,2 & 48,1 & 9,2 \\
\hline Ephedra antisyphilitica.......... & Pousses vertes & 6,9 & 1,5 & 33,7 & 51,5 & 6,3 \\
\hline Hibiscus esculentus ..... & Graines & 24,7 & 19,7 & 30,1 & 20,6 & 4,9 \\
\hline Mimosa fragrans ..... & Feuilles & 20,6 & 3,5 & 21,2 & 47,7 & 7,1 \\
\hline Morus Inicrophylla ..... & id. & 15,9 & 5,6 & 12. & 50,4 & 16. \\
\hline Opuntia atrispina $\ldots .$. & Raquettes sans épines & 7,4 & 1,2 & 11,5 & 58,3 & 21,6 \\
\hline Opuntia castillae .... & Tiges & 7,9 & 2,2 & 11,8 & 53,8 & 24,3 \\
\hline Prosopis chilensis . . . . . . . . . . & Gousses & 13,7 & 1,9 & 27,9 & 52,6 & 3,9 \\
\hline Pueraria thunbergiana... & Plante entière & 13,3 & 4,2 & 31,1 & 39,9 & 11,6 \\
\hline Quercus virginiana & Feuilles & 19,2 & 1,2 & 24,2 & 50,4 & 5 \\
\hline Rhus microphylla............. & id. & 16,4 & 2,6 & 11,2 & 64,8 & 5 \\
\hline Yucca glauca ....... & Feuilles-Tiges & 7,6 & 2,1 & 40,4 & 41 & 8,8 \\
\hline
\end{tabular}

